\title{
A Survey Study of the Current Challenges and Opportunities of Deploying the ECG Biometric Authentication Method in IoT and 5G Environments
}

\author{
Ahmed Shdefat ${ }^{1}$, Nour Mostafa ${ }^{1}$, Louai Saker ${ }^{1}$, and Ahmet E. Topcu ${ }^{1}$ \\ ${ }^{1}$ IST/TNT Department, College of Engineering and Technology, \\ American University of the Middle East, Egaila, Kuwait
}

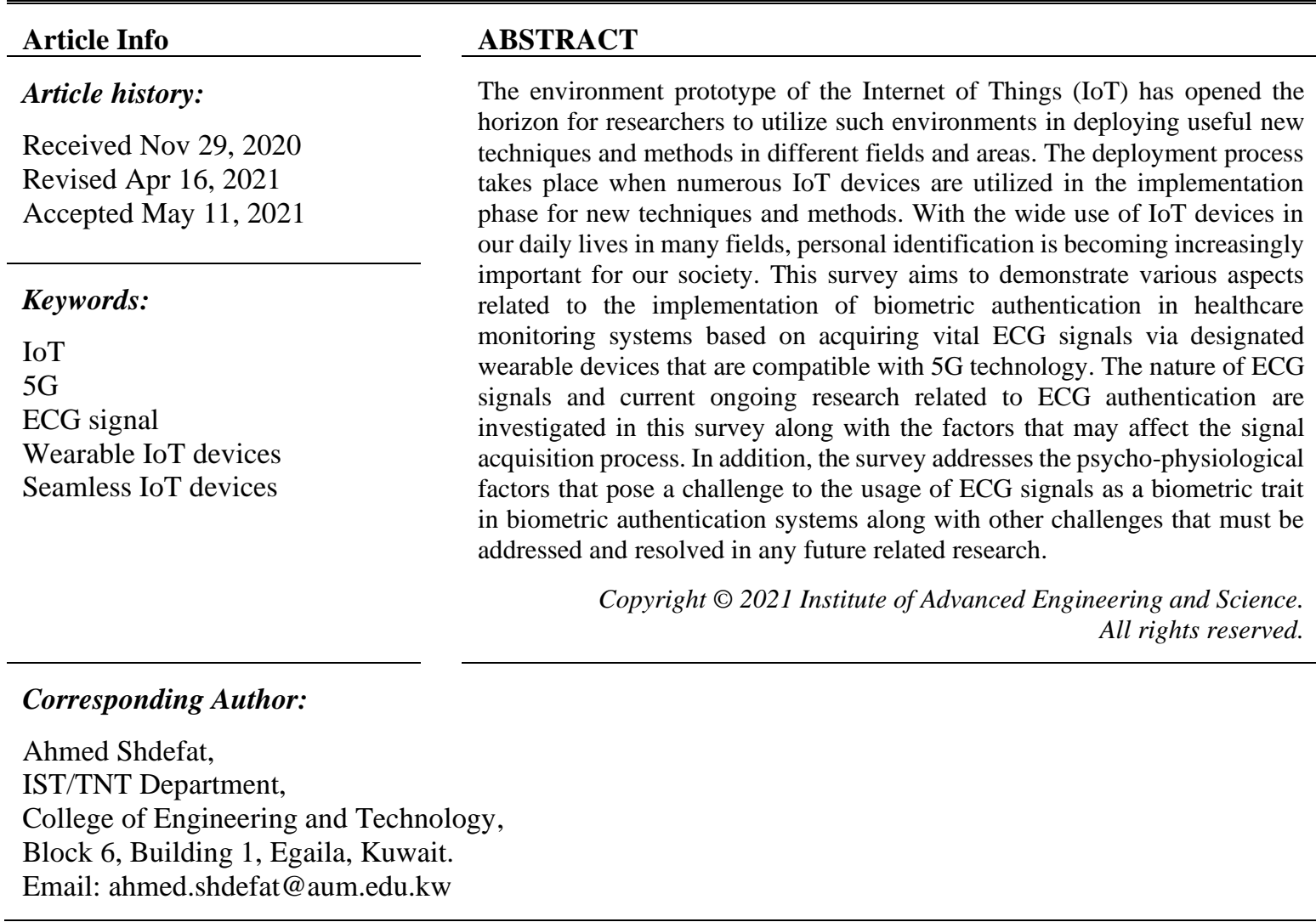

\section{INTRODUCTION}

There has been a recent rapid increase in data generation from users and Internet of Things (IoT) devices such as sensors, controllers, and others. These devices are used as smart objects that communicate with each other. The IoT is a technology that allows the communication of smart things through a heterogeneous and decentralized network [1]. The IoT fundamentally depends on three paradigms: the Internet (network), things (devices), and data (conveyed knowledge) [2]. The development of IoT platforms leads to smart homes, smart cities, smart agriculture, driverless cars, and systematized factories, among other possible applications.

People now generally need to adapt to this new paradigm after rapid changes in their lifestyles. Most IoT applications run on cloud environments that provide highly efficient performances. Many different IoT platforms exist to process data effectively on the cloud. For example, the Amazon IoT (AWS IoT) platform provides scalable, secure, and intelligent services by connecting many devices through cloud services such as AWS Lambda, Amazon Kinesis, and Amazon S3 [3]. The Azure IoT platform provides communication with services defined in the cloud environment [4]. Correspondingly, the device manager and protocol bridges (MQTT and HTTP) are two major components of the Google IoT platform, and by collecting temperature measurements from the surrounding environment using IoT devices, for example, the collected event data can be transferred to the cloud using telemetry [5]. 
During the COVID-19 pandemic, IoT devices such as smartphones with embedded sensors using machine learning techniques have intelligently helped track COVID-19 patients using real-time data utilization [6]. Lampropoulos et al. [7] presented detailed survey results regarding IoT industrial applications that affect industrial developments and trends. The number of smart factories, for example, is expected to increase dramatically in the upcoming years with new trends of manufacturing that depend on smart IoT devices, inevitably moving in the direction of modeling human-free and robot-controlled factories. Another report [8] indicated that healthcare services will be more vigorous on the heels of the COVID-19 pandemic. Robust services in healthcare systems may be provided by IoT-based solutions such as remote patient control, robots, and others, especially in quarantined hospitals. Data generated from these devices can then be conveyed to medical systems in order to handle situations and obtain accurate results using machine learning [9].

At the same time, the number of smartphone users is increasing massively every year. According to one report [10], recent statistics show that the number of smartphone device users exceeded 3.5 billion in 2020 and is expected to surpass 3.8 billion in 2021. At this rate, the current mobile network infrastructure using fourth generation (4G) or Long-Term Evolution (LTE) technology will not be able to support all of the newly produced devices with IP addresses to be attached to the network to communicate actively due to the limited nature of the currently used communication protocol in the networking field, which is IPV4 [11]. In addition, as more devices are connected to the network and actively communicating, more latency and delay will occur, causing low-quality telecommunication services for users [12], [13].

Considering the fundamental point of the IoT, which makes everything connected, including heterogeneous things like the future's high volume of smartphone devices, computing devices, appliances, sensors, and objects, a demand is being created for infrastructure with enhanced data rates, more bandwidth, better capacity, minimized latency, and better quality of service [14]. Pervasive interactions between heterogeneous objects such as smart autonomous cars, sensors, appliances, and smartphones within the environments of smart homes, smart cities, and smart grids are triggering the issue of channel access for various heterogeneous objects via different applications, such as medical monitoring applications using vital signs and security applications using biometric authentication methods [15].

The current LTE or 4G infrastructure will be crippled under this load and will not be able to meet the vital IoT demands of making everything within the IoT environment connected through a reliable network, especially while attempting to handle the produced data traffic that will result from the interactions between these connected devices [11], [12]. In order to meet these demands and build a stable and reliable IoT environment, telecommunication companies, researchers, and educational institutions have started to explore alternative advanced technology infrastructures to meet the IoT demands [14]. The fifth generation (5G) of wireless communications technologies supporting cellular data networks has emerged as a proposed solution to resolve $4 \mathrm{G}$ limitations in meeting the demands of the IoT environment infrastructure. Therefore, $5 \mathrm{G}$ now represents the backbone of the IoT environment. In 5G architecture, there are three frequency spectrums: millimeter waves, mid-band, and low-band. The millimeter wave download speed ranges from 1 to 2 Gbit/s with frequencies above $24 \mathrm{GHz}$ and below $72 \mathrm{GHz}$; the optimal frequency is $28 \mathrm{GHz}$. In the mid-band $5 \mathrm{G}$ case, download speeds are in the range of 100-400 Mbit/s with frequencies from 2.4 to $4.2 \mathrm{GHz}$. Finally, the lowband $5 \mathrm{G}$ frequency spectrum is identical to the one currently used for $4 \mathrm{G}$ [16]. This survey investigates the different biometric authentication methods deployed within the 5G IoT infrastructure in general and the electrocardiogram (ECG) biometric authentication method in particular.

Biometrics are defined as biological measurements or recordings of physical characteristics, such as fingerprint mapping, iris scanning, face recognition, behavioral characteristics, and voice recognition, which are used by an authentication system to identify and verify individuals and/or objects [17]. Biometric authentication systems are growing in complexity, as they have to handle ever-greater numbers of wearable devices, which are becoming an extended part of our bodies. Therefore, there is an increasing demand for an automatic and reliable authentication system. In the last decades, the above-mentioned biometric systems have been considered as reliable paradigms [18],[19]. Biometric data types vary, but the most popular types are explained below.

The current trends for smart devices, including smartphones, wearable devices, and voice automatic assistant services, involve using voice interactions rather than traditional touch interfaces to perform many tasks including, but not limited to, making phone calls, sending messages, checking emails, performing banking services, and using driver assistant services [20]. In addition, vocal features may be used in voice biometric authentication. However, most of the current applications use this mechanism as a voice assistant, not as an authentication method, because it is difficult to secure, in addition to its vulnerability and exposure to many threats [21], [22].

Fingerprinting is used as a biometric method to identify the unique patterns of ridges and valleys on fingers. Sir William Herschell was the first to note the value of fingerprints for identification [23], [24]. From the beginning of the twentieth century, the fingerprint was acknowledged as the most permanent biometric trait 
due to characteristics such as its uniqueness and permanency over time, with fingerprinting being a very fast technique, cheap, accurate, and acknowledged by the legal community as reliable. Many organizations use biometric fingerprint authentication in the workplace as a time attendance system [25]. The massive growth of smartphone users has also encouraged developers and researchers to use fingerprints in other applications such as online banking[26], E-healthcare [27], and commercial transactions [28].

Iris recognition is used as a biometric method to identify the unique pattern of a person's iris. As reported by Shirke and Rajabhushnam [29], the human iris has 256 patterns to be used as training images in biometric databases. Sensors are used on the infrared spectrum to collect these images. Iris biometrics share similar characteristics with fingerprints in terms of uniqueness, reliability, and permanency over time [30]. By extracting the iris region in terms of the inner and outer boundaries of the image, iris recognition systems determine the unique textures of individual irises. Iris biometric authentication systems are used in many applications such as premise access control, banking transactions, identification screening systems at airports, and many more [31].

Face recognition is also used as a biometric method to identify individuals by their faces. Simple geometric models were used in the early stages of face recognition systems. Nowadays, however, engineering principles are implemented, which has turned face recognition into a science in itself [32], allowing it to be used for both identification and verification. Face recognition has become one of the most popular biometric systems since it can be performed in unconstrained environments while providing strong discriminative features for recognition [33]. From the computer's perspective, it is the extraction of facial texture and shape. Face recognition has been used in many applications and systems such as security systems, video analytical systems, smart shopping, automatic face tagging, and device access control.

In recent years, electrocardiogram (ECG) was introduced as another biometric authentication method to overcome the drawbacks of the above biometric authentication methods, as those methods can easily be dodged [34], while ECG signals are very hard to duplicate [35]. ECG recognition systems take the ECG signal from a person as input and the output is the identification of that person. With the increasing number of IoT environments and applications such as smart cities, smart health, smart homes, energy management, transportation, elder care, and environmental monitoring, researchers and developers are focused on both security and privacy, which have become more challenging. This technology has become more ubiquitous due to the increasing number of wearable devices. Therefore, new biometric authentication technologies are being introduced, such as ECG, to offer more robust modalities.

Figure 1 shows the proportion of applications using selected types of biometric technologies in 2018 according to German and Barber [36]. Statistical results show that fingerprinting is the most common type of technology for authentication as $40 \%$ of applications used fingerprint readers. Face recognition is also popular as $15 \%$ of applications used facial recognition technology while $13 \%$ used iris scanning technology.

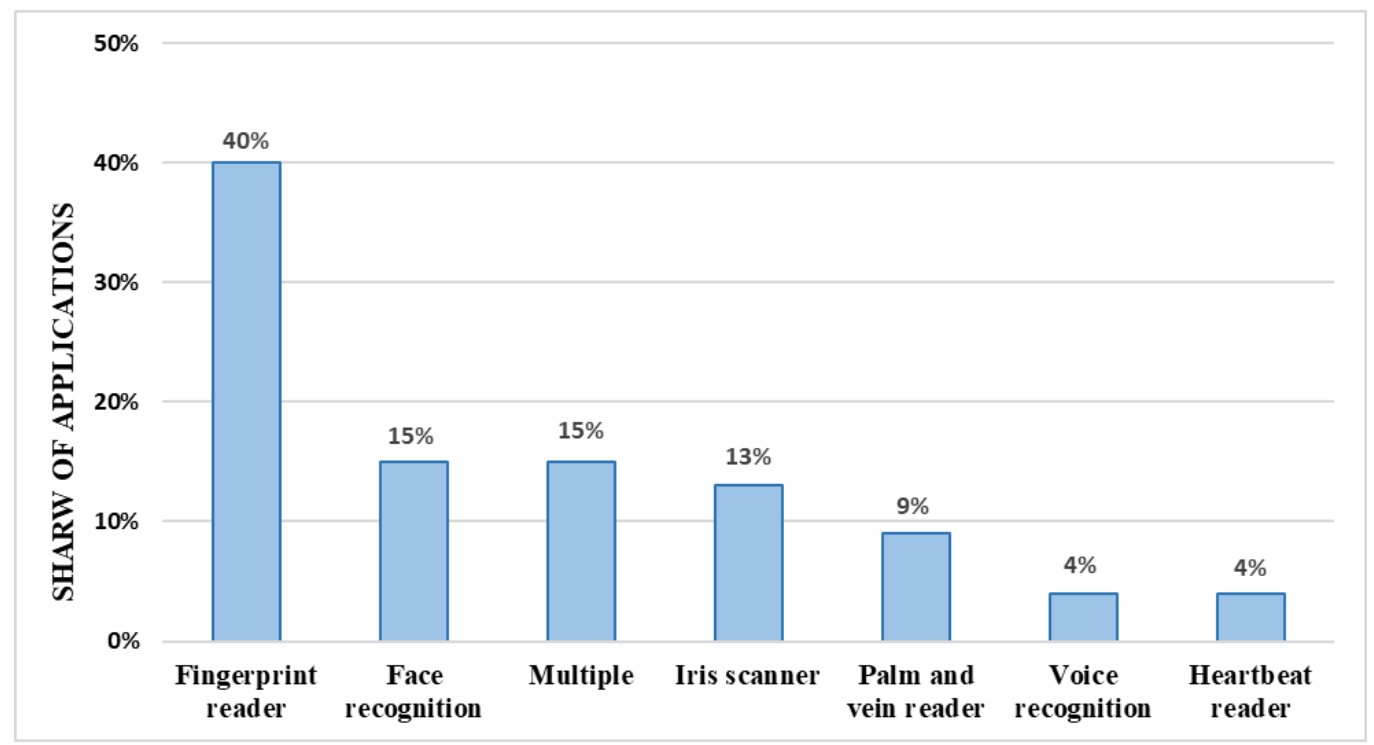

Figure 1. Biometric technologies used in applications in 2018

According to one report [37], the global biometric technology market was valued at 17.16 billion USD in 2018 and is expected to reach approximately 59.43 USD billion by 2025, growing at a compound annual growth rate of $19.42 \%$ between 2019 and 2025. The deployment of mobile biometrics is growing rapidly [38] and this is now considered a robust authentication method for virtually all mobile devices and smartphones 
[39], [40]. Table 1 compares some biometric technologies in terms of different aspects such as accuracy, performance, cost, measurability, and security level. It also shows the drawbacks of each type of technology.

Table 1. Comparison of different authentication technologies [37], [39]

\begin{tabular}{ccccccc}
\hline Technology & Accuracy & Performance & Cost & Measurability & Security Level & Drawbacks \\
\hline Fingerprinting & Medium & Medium & Low & Medium & Low & Temperature, humidity, injury \\
Iris Recognition & High & High & High & Medium & Medium & Illumination, injury, resolution \\
$\begin{array}{c}\text { Face } \\
\text { Recognition }\end{array}$ & Low & Medium & High & High & Low & Illumination, injury, resolution \\
ECG & High & High & High & Medium & High & Psychological and physical state sensitivity \\
\hline
\end{tabular}

In the next section, a more extensive review of related ECG studies is presented. Section 3 reviews the signal methodologies. Section 4 describes the factors affecting ECG signals. Section 5 explains the deployment of ECG within the 5G IoT environment. Section 6 provides details on signal acquisition approaches. Section 7 highlights the challenges and opportunities of wearable and seamlessly integrated devices. Section 8 presents the ECG databases. Section 9 describes the preprocessing and feature extraction of ECG signals. Section 10 presents 5G IoT architecture and infrastructure. Section 11 presents ECG deployment opportunities and challenges and Section 12 provides a conclusion and suggestions for future work.

\section{LITERATURE REVIEW}

Kamble and Birajdar [41] proposed an ECG recording and monitoring framework designed to send ECG data directly to IoT cloud storage using Wi-Fi, in addition to using SD cards for offline storage. The proposed architecture collects real-time ECG signals using a wearable monitoring node with three electrodes. A thin-film transistor LCD is used to display the real-time ECG signals for patients and doctors. A web interface was developed on an IoT cloud site to view the ECG signals, which can be accessed by smartphones, desktop computers, and laptops.

The architecture proposed by Chen et al. [35] offers a number of physiological measurement sites to measure individuals' physical and psychological statuses via an application stored on a smartphone and used as a cloud monitor of psychological status, combined with an ECG authentication mechanism. The proposed model uses a 1-lead ECG instrument instead of a 12-lead ECG instrument as it is more suitable and convenient for biometric authentication in real-time IoT applications. The proposed model uses sensors to measure and transmit physiological information, which is sent later to the cloud for data management and analysis. A pulsemeasuring device is used to filter noise and amplify pure signal, and then it is used for authentication when the user logs in to the app as an ECG biometric system. A backpropagation neural network technique is used to obtain the recognized object. This architecture yielded a successful ID recognition rate of $92.86 \%$.

Pinto et al. [40] discussed and reviewed the state of the art among available public ECG collections for biometrics research, signal processing, and datasets in addition to the proposed methods and techniques used for acquisition. Their survey also discussed the ECG biometrics algorithms used for signal processing operations, in addition to denoising, feature extraction methods, fiducial detection, and signal segmentation. They briefly addressed the approaches for on/off-the-person signals and recommended efforts to improve the denoising techniques, as more noise will survive with the rise of off-the-person, wearable, and seamless devices. This survey concluded that as noise increases, a combination of fiducial and non-fiducial methods is recommended to reflect the best conditions offered by both method types, such as the high performance offered by fiducial methods and the variability and robustness to noise offered by non-fiducial methods. Furthermore, these authors discussed the state of the art of current ECG biometrics algorithms for decision methods and the most common decision algorithms with proven performance superiority were reviewed, such as support vector machines (SVMs), artificial neural networks (ANNs), and deep neural networks (DNNs). Security issues were covered very briefly in the survey, as there is still a long way to go before personal information is stored securely in ECG biometric systems. The topics covered in this survey were thus extensive; however, considering the rise of IoT devices in our daily lives and the need to apply personal identification to these devices, the survey did not cover crucial topics such as 5G and IoT or their integration with ECG biometric systems, which will be addressed in this survey.

According to El Zouka and Hosni [42], in order to preserve the quality of public healthcare systems targeting elderly people, there is a pressing need to implement new models for information management and communication. Hence, wireless sensor network technology can be used in critical situations during the process of collecting patient's vital signs, and, more specifically, ECG signals. The authors recommended employing such technology in the implementation of new models. 
In brief, implementing a healthcare system using a wireless medical sensor network (WMSN) for the monitoring process along with radio frequency identification (RFID) body sensors will make the system capable of transmitting patients' medical status data wirelessly to a local workstation. The main drawback of such a system is that the data received via WMSN are not utilized properly due to deficiencies in network architecture, storage, security, and protocol standards [42].

Moosavi et al. [43] identified the vital requirements for a common IoT healthcare system that will make it possible to obtain a robust system security solution. They used an efficient and well-protected verification technique for healthcare IoT devices, implemented by applying robust and secure mobility-enabled end-to-end communication along with certificate-based Datagram Transport Layer Security (DTLS). The technique of producing ECG-based cryptographic keys was used for medical sensor devices to trigger the designed end-to-end security scheme and the DTLS technique in the handshake event along with the session resumption mechanism for communications. Based on these authors' conclusions, all of these techniques function 1.8 times faster on average than existing similar key generation approaches.

The survey undertaken by AbdElnapi et al. [44] provided a summary of the needed technologies for healthcare services, a list of medical sensors commonly used in IoT research for ECG signals, and the current novel healthcare projects being conducted within the IoT field. Their survey focused on the employment of current IoT technologies in the healthcare field and presented a conclusion about the current stage of development in which these IoT technologies have been deployed effectively in healthcare and related issues. The core contribution of this survey lay in its examination of how the IoT environment could have a direct impact in enhancing and improving people's quality of life. It listed and summarized state-of-the-art IoT technologies for healthcare such as cloud computing, RFID sensors, big data analysis, WSN, Wi-Fi, and Bluetooth. In addition, it tackled the current and possible future challenges facing the IoT-based environment in healthcare systems. These authors urged that the existing performance of healthcare systems within the IoT environment must be improved to meet the emerging demands [44].

In healthcare systems, IoT-enabled devices are being used for ECG monitoring using Wi-Fi, Bluetooth, and Zigbee technologies. Accumulated data from IoT devices are transferred to the cloud through wearable nodes using MQTT protocols. The model proposed by Yang et al. [45] shows that ECG data may be easily transferred from IoT devices to cloud environments and can be used to analyze the results effectively.

Depending on the specific healthcare problems, Wi-Fi, Bluetooth, or Zigbee technologies may be used for effective data collection and highly accurate results. The new model proposed by Yang et al. [45] can be used to collect ECG data via sensors through a cloud infrastructure. In addition, data cleaning techniques and different model analyses were exploited to establish a disease warning system for patients at home.

Many companies and researchers are now making explicit efforts to achieve 5G technologies that provide heterogeneous architectures in order to respond to the growing demand for network capacity and seamless links for distributed radio access technology to attain fast and effective communication. One of the important issues here is to build different effective types of architecture to provide fast and reliable data communications for the environments of medical devices using $5 \mathrm{G}$ technologies. Shakhakarmi described different types of device-to-device (D2D) communications for heterogeneous 5G architectures for wearable medical devices [46]. It was also argued that designing wearable ECG system-on-chip architecture and using EEG for low-range D2D communications will facilitate the effective optimization of wireless data transmissions.

Islam et al. [47] presented detailed survey results regarding new developments in IoT-based healthcare technologies, including the new trends and opportunities in IoT-based healthcare, security and privacy issues, and the monitoring of ECG using IoT applications. Their survey categorized IoT healthcare networks into three parts: topology (the organization of different elements), architecture (the specification of the physical IoT healthcare elements), and platform (the framework for network and computing). They also provided a list of smartphone applications for general health monitoring. According to their survey, the increasing number of heart rate monitoring applications and mobile applications such as Cardiax Mobile ECG and ECG SelfMonitoring indicate the importance of IoT-based ECG monitoring systems.

ECG biometric authentication algorithms offer better security mechanisms compared to popular biometric techniques like fingerprinting that can be spoofed by an attacker who captures fingerprints left by users on objects. Arteaga-Falconi et al. [48] proposed and designed an ECG-based authentication algorithm that uses a hierarchal scheme with a short acquisition time for mobile authentication. The proposed algorithm acquired ECG signals by using only two electrodes of the mobile device to gain access, and the obtained results showed that the algorithm needs 4 seconds of signal acquisition to identify unknown individuals with a $1.41 \%$ false acceptance rate and $81.82 \%$ true acceptance rate.

Tan and Chung [49] proposed an effective authentication mechanism between wireless entities in order to provide preliminary protection for the interactions of wireless body area networks (WBANs). They developed a secure certificateless biometric authentication and group key management for WBAN scenarios. 
The user's smart device takes on the role of a personal controller in a WBAN structure. The proposed mechanism is classified into different phases including the offline registration phase, ECG feature extraction phase, authentication phase, group key distribution phase, and group key updating strategy.

From an embedded computing perspective, Samie et al. [50] summarized the requirements of IoT technologies that offer improvements in some application areas like ECG healthcare and smart homes. They discussed the architecture of IoT embedded devices with the main components that are responsible for data acquisition, processing, storage, and transmission. They described the available resources, the characteristics of wireless technologies, and network latency for different computation layers starting from IoT embedded devices such as ECG sensors and continuing up to cloud servers.

Maqbool et al. in [51] offered a real time patient monitoring system which utilize Message Queuing Telemetry Transport (MQTT) in handling real time ECG signals' data and operating IoT environment. The proposed system provides health care specialists the ability to check the real time patient ECG data via a mobile application or the web. The proposed system was tested over both LAN and WAN networks' infrastructures. The authors illustrated the system advantages over serving patient in rural area, achieving $90 \%$ accuracy handling ECG data, in addition to the ability to detect ECG signal abnormality and the system expandability nature to have more than a few extra health parameters.

Wang et al. in [52] examined the user authentication based on ECG biometric method for IoT edge devices. Their proposed model deployed the Convolutional Neural Network (CNN) for user authentication using deep learning technique in IoT environments. The authors had tested 290 ECG samples from physionet database and their proposed model achieves $99.63 \%$ authentication accuracy using CNN along with deep learning technique. Furthermore, the proposed model achieves $98.88 \%$ authentication accuracy in the process of optimizing the time and accuracy factors over processing complex data sets.

C. Liu et al. in [53] developed an IoT-based wearable ECG SmartVest to monitor ECG signal in real time and to detect cardiovascular diseases in early stage. The proposed system combines the multiple SQI and SVM-based machine learning to classify the signal quality of ECG signal in several situations. The authors use the cloud platform to manage cloud ECG database, and implement artificial intelligence in cloud computing which process ECG data to provide a disease prediction and diagnostic. The SVM-based model achieves an average accuracy of $97.9 \%$ for acceptable ECG segments in real wearable ECG monitoring.

L. Xiaolin et al. in [54] proposed a deep-learning-based Convolutional Neural Network (CNN) model for heartbeat classification using a single lead ECG. The proposed model classifies an ECG signal into five classes using the MIT-BIH Database. The proposed model uses the Synthetic Minority Oversampling Technique (SMOTE) algorithm to balance the training data by augmenting it with a synthetic data. The obtained results can achieve an overall accuracy of $98.12 \%$ and it reflects a real-world performance. Table 2 represent a comparison between the different commonly used methods in the accuracy factor for processing the ECG single within the IoT environments.

Table 2. Comparison between the commonly used methods in the related works in term of the ECG handling accuracy in IoT environments

\begin{tabular}{lll}
\hline Related work & Method & Accuracy \\
\hline Maqbool et al. in [51] & $\begin{array}{l}\text { Real-Time PMS Architecture using a } \\
\text { broker of MQTT }\end{array}$ & $90 \%$ \\
Wang et al. in [52] & $\begin{array}{l}\text { A Convolutional Neural Network (CNN) } \\
\text { based deep learning technique }\end{array}$ & $98.88 \%$ \\
C. Liu et al. in [53] & $\begin{array}{l}\text { A combination of multiple signal quality } \\
\text { indices (SQI) and Support vector machine } \\
\text { (SVM)- based classification. }\end{array}$ & \\
& A deep-learning-based CNN model for & $98.12 \%$ \\
L. Xiaolin et al. in [54] & & \\
\hline
\end{tabular}

\section{SIGNAL MORPHOLOGY}

Basically, the heart consists of four main parts, called chambers, which are in a cone-like muscular pump format in the center of the chest, located between the lungs and below the sternum [55]. The heart's function is to supply the tissues with the needed nutrients and oxygen and ensure the excretion of carbon dioxide and metabolic waste through the lungs and the kidneys via the blood circulation process [56], [57]. As shown in Figure 2, the atria and ventricle chambers are separated by fibrous valves, which makes them electrically isolated by the non-conductive tissue that separates them [58]. 


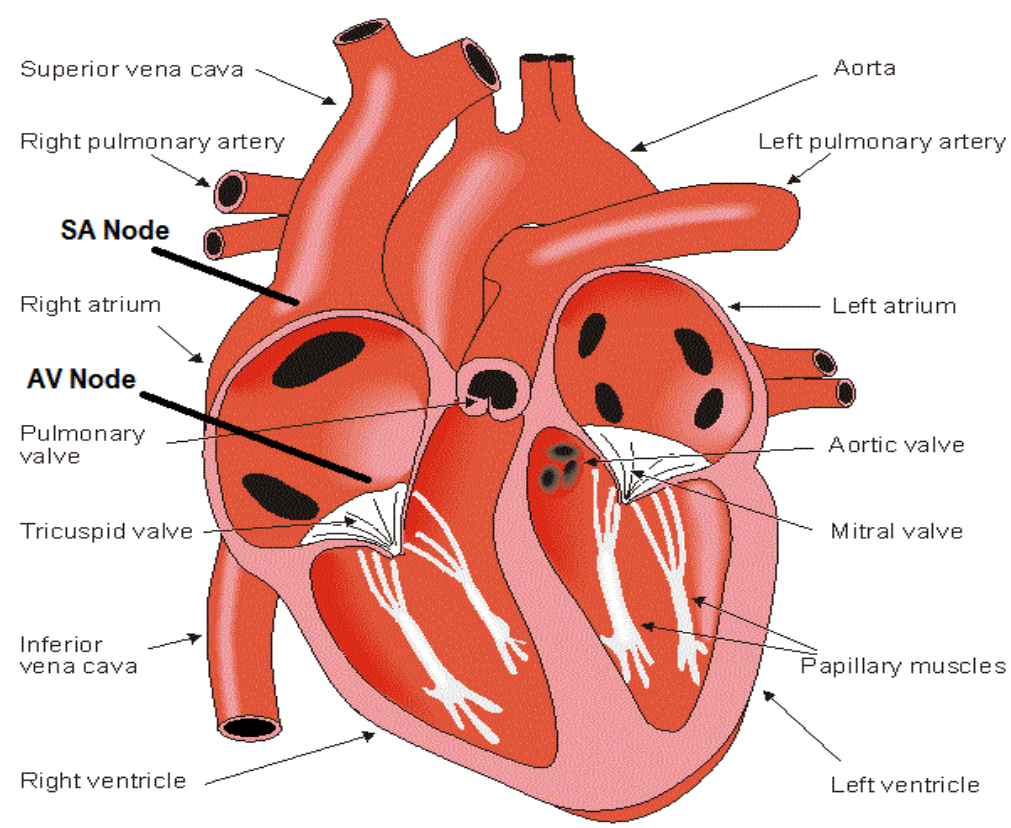

Figure 2. Heart physiology

The blood circulation of the heart begins when deoxygenated blood is received through the superior and inferior vena cava, pouring into the right atrium and right ventricle by passing through the tricuspid valve. The blood then moves to the pulmonary valve, towards the left and right pulmonary artery, located near the lungs. At this point, the blood removes carbon dioxide and absorbs oxygen.

The oxygenated blood travels back to the heart through the pulmonary veins, pouring into the left atrium and left ventricle while passing the mitral valve. Subsequently, the blood will travel through the aortic valve to the rest of the body when the left and right ventricles are contracted, as shown in Figure 3. In other words, the chambers of the heart alternatively contract and relax in a rhythmic cycle [59].

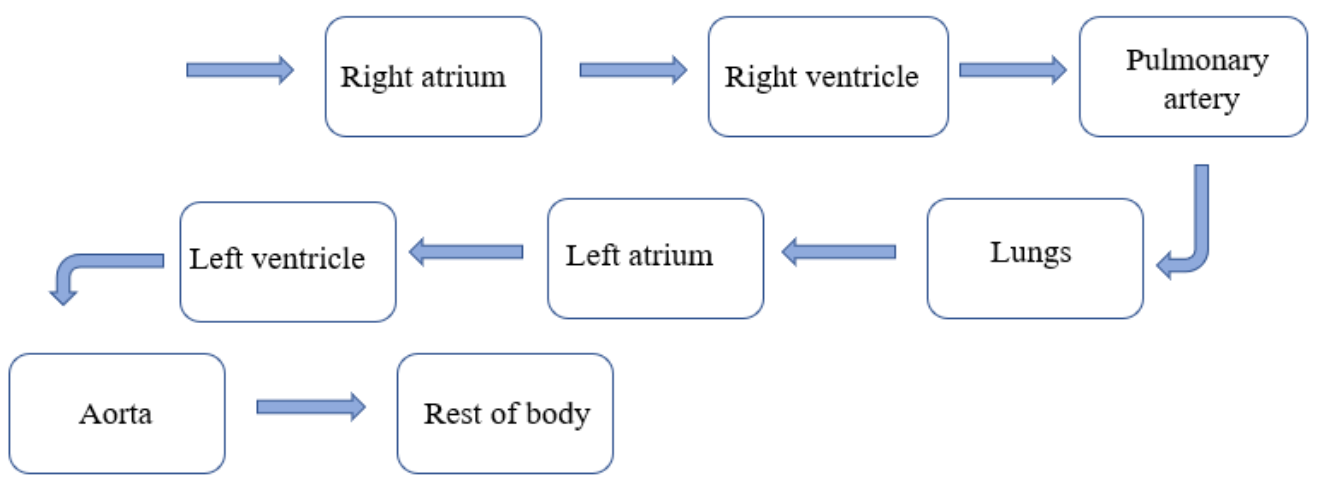

Figure 3. Blood circulation

When the heart pumps the blood out via the arteries, the heart is in a contraction state called systole. On the other hand, when the heart is in a state of relaxation, called diastole, blood fills the chambers. A complete sequence of systole and diastole constitutes what is known as a cardiac cycle. The heart rate is the measurement of cardiac cycle frequency, representing a value of number of beats per minute [56].

The sinoatrial (SA) node is a part of the heart that controls the rhythm of contractions and triggers the cardiac cycle like a natural pacemaker by generating electrical pulses and spreading them throughout the heart along conducting tissue pathways, as illustrated in Figure 2. A normal heart rate is between 60 and 100 beats per minute. When the signal moves from the SA node through the right and left atria, it causes contraction effort, or depolarization, and the blood moves into the ventricles [60]. This depolarization event takes place when the primary electrical vector is directed from the SA node towards the atrioventricular (AV) node, which basically spreads from the right atrium to the left atrium. This event creates the P wave of ECG signals [61]. Table 3 provides the normal durations of waves, intervals, and segments in ECG signals [57], [62]. 
Table 3. Normal durations of waves, intervals, and segments in ECG signals [57]

\begin{tabular}{|c|c|c|}
\hline Feature & Description & Duration \\
\hline $\mathrm{RR}$ interval & $\begin{array}{l}\text { Interval between an } \mathrm{R} \text { wave and the next } \mathrm{R} \text { wave; normal resting heart } \\
\text { rate is between } 60 \text { and } 100 \mathrm{bpm}\end{array}$ & 0.6 to $1.2 \mathrm{~s}$ \\
\hline P wave & $\begin{array}{l}\text { During normal atrial depolarization, the main electrical vector is } \\
\text { directed from the SA node toward the AV node and spreads from the } \\
\text { right atrium to the left atrium; this turns into the ECG P wave }\end{array}$ & $80 \mathrm{~ms}$ \\
\hline PR interval & $\begin{array}{l}\mathrm{PR} \text { interval is measured from the beginning of the } \mathrm{P} \text { wave to the } \\
\text { beginning of the QRS complex }\end{array}$ & $\begin{array}{l}120 \text { to } \\
200 \mathrm{~ms}\end{array}$ \\
\hline PR segment & $\begin{array}{l}\text { The PR segment connects the } \mathrm{P} \text { wave and QRS complex; the impulse } \\
\text { vector is from the AV node to the bundle of His to bundle branches } \\
\text { and then to the Purkinje fibers }\end{array}$ & $\begin{array}{l}50 \text { to } \\
120 \mathrm{~ms}\end{array}$ \\
\hline QRS complex & $\begin{array}{l}\text { The QRS complex reflects the rapid depolarization of the right and } \\
\text { left ventricles; they have a large muscle mass compared to the atria, } \\
\text { so the QRS complex usually has a much larger amplitude than the } \mathrm{P} \\
\text { wave }\end{array}$ & $\begin{array}{l}80 \text { to } \\
120 \mathrm{~ms}\end{array}$ \\
\hline ST segment & $\begin{array}{l}\text { The ST segment connects the QRS complex and T wave; it represents } \\
\text { the period when the ventricles are depolarized and it is isoelectric }\end{array}$ & $\begin{array}{l}80 \text { to } \\
120 \mathrm{~ms}\end{array}$ \\
\hline $\mathrm{T}$ wave & $\begin{array}{l}\text { The } \mathrm{T} \text { wave represents the repolarization (or recovery) of the } \\
\text { ventricles; the interval from the beginning of the QRS complex to the } \\
\text { apex of the } \mathrm{T} \text { wave is referred to as the absolute refractory period }\end{array}$ & $160 \mathrm{~ms}$ \\
\hline ST interval & $\begin{array}{c}\text { The ST interval is the interval between ventricular depolarization and } \\
\text { repolarization }\end{array}$ & $320 \mathrm{~ms}$ \\
\hline QT interval & $\begin{array}{l}\text { The QT interval is measured from the beginning of the QRS complex } \\
\text { to the end of the T wave }\end{array}$ & $\begin{array}{l}\text { Up to } 420 \mathrm{~ms} \\
\text { at a heart rate } \\
\text { of } 60 \mathrm{bpm}\end{array}$ \\
\hline
\end{tabular}

Heart cells are polarized when the heart status is in rest, and this indicates that there are no electrical actions happening. The heart status is considered to be depolarized during the systole period and repolarized during the diastole period. In a standard ECG wave, depolarization starts from the P wave up to the midpoint of the ST segment. In time, repolarization begins from the T wave until the very end of the signal, as shown in Figure 4 [57], [63].

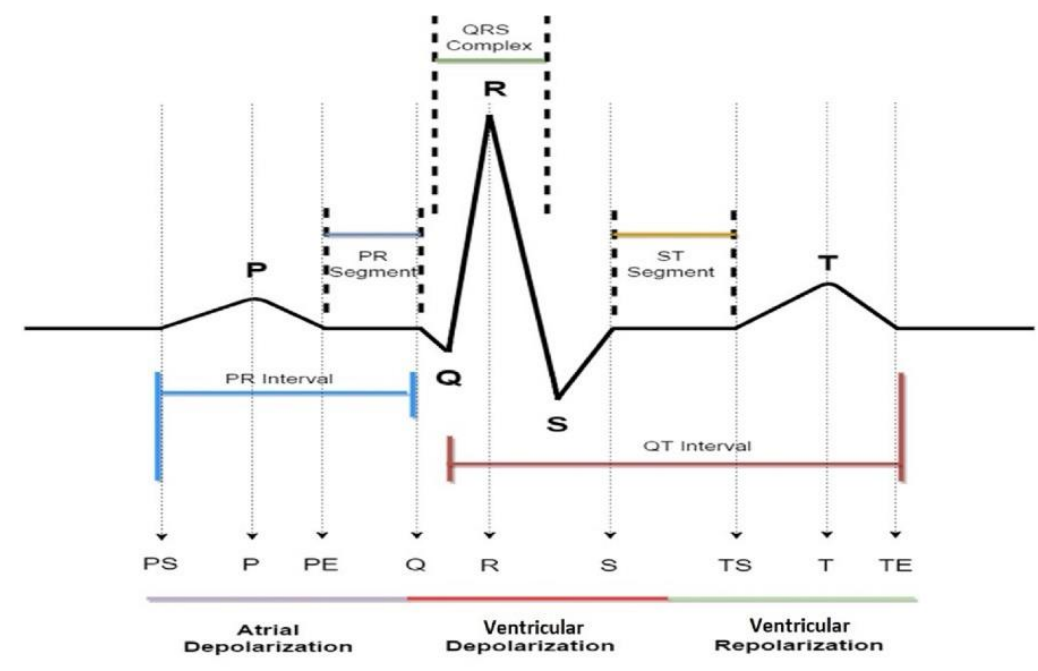

Figure 4. ECG signal physiology

In a standard ECG signal, the features are classified as either characteristic-based features or waveforms. Characteristic-based features are straightforward in terms of acquisition and processing because, to set off the extraction activity, we only need knowledge of one of the ECG complexes that crisscross the fiducial points in the signal. ECG fiducial points are represented by points PS, P, PE, Q, R, S, TS, T, and TE, which crisscross over the signal, and these crisscrossing points intersect with the ECG signal's peaks and boundaries as shown in Figure 4 [57], [64], [65]. In an ideal ECG signal, there three peaks and six boundaries, as illustrated in Figure 4 [63].

In most ECG-related research, the targeted features to be obtained are angle, slope, amplitude, and temporal duration. Additional intensive processing activities must be applied to the targeted features to get the 
discriminative characteristics [65]. In the presence of noise and when using the fiducial points to obtain the characteristic-based features, problems arise in identifying the boundaries.

The Fourier coefficient, autocorrelation, phase space reconstruction, and wavelet coefficient are considered as the coefficient values to be processed in the waveform-based type of classification in order to obtain the signal features. In the feature extraction phase using the waveform method, more than one ECG complex needs to be utilized. The advantage of using more ECG complexes in the feature extraction phase is that boundary detection difficulties in the presence of noise can be overcome because there is no need for using fiducial point detection [63], [65].

Heart rate variability (HRV) is commonly described as a measurement of the beat-to-beat variation in the interval time of R-R peaks between consecutive heartbeats, evaluating physiological variation. HRV analysis of heart activities reflects the state of the autonomic nervous system and is usually done by one of two types of methods: linear and nonlinear methods [66].

\section{FACTORS AFFECTING ECG SIGNALS}

Portable systems are currently used in many IoT devices and applications to monitor heart activity and record ECG signals to be used for biometric authentication in addition to diagnostic and treatment applications. ECG signals are often contaminated with noises during the recording process, which will affect their accuracy and valuable information. There are two types of interference that can affect the signal, namely environmental and non-environmental factors, such as noises coming from muscle artifacts, baseline wander, noise from other electrical instruments, high-frequency electrical noises, electromyography interference, and electrode motion [67], [68]. Due to the low frequency, high impedance, and low signal level of ECG signals, the signal preprocessing stage is central in any automatic ECG signal processing task [69]. The main purpose of the preprocessing stage is filtering the ECG signal to be resistant to interference by using a denoising technique to suppress artifacts and background noise.

Identification of noises in ECG segments is of utmost important to achieve noise-free ECG signals. Various denoising techniques were proposed in the earliest days of ECG denoising research, such as filter banks [70], low-pass filters [71], and adaptive filters [72]. Signal quality assessment (SQA) was one of the first techniques used as an initial stage of this process by analyzing the signal quality and then making a decision to accept or reject the input signal [73]. This process is semi-automated because SQA requires a human operator to make the decision of rejecting the corrupted signals, and it also has higher levels of time consumption and error during measurements. Therefore, researchers moved on to automated techniques for most decisionmaking systems [68]. Many extended SQA techniques have been proposed to transform the SQA from a semiautomated technique into a fully automated one, such as simple thresholding, fiducial point detection, machine learning approaches, the transform domain, morphological change detection, and signal decomposition [74].

Kalman filters [75] and the discrete wavelet transform are the most exploited approaches proposed for ECG noise reduction using the polar variants of the morphological ECG dynamic model introduced by McSharry et al. [76]. Some existing ECG denoising techniques have involved nonlinear projective filtering [77] and Bayesian filtering [78]. There are many existing ECG denoising techniques proposed based on time domain signal decomposition techniques [78][82]. One of the most efficient ECG noise filtering techniques is the non-local means algorithm [83][85]. Most state-of-the-art ECG denoising techniques have their own individual shortcomings. However, the main issue with the existing techniques is their ability to denoise different noises, as most of them were proposed to denoise a maximum of one or two noisy conditions during the recording process of ECG signals. This needs to be improved in the future [86].

\section{DEPLOYING ECG WITHIN the 5G IoT ENVIRONMENTS}

According to many researchers, such as Shaown et al. and Santos et al. [87][88], IoT-based ECG monitoring systems may be categorized into the three interactive parts of ECG sensing network, IoT cloud, and graphical user interface (GUI), as shown in Figure 5.

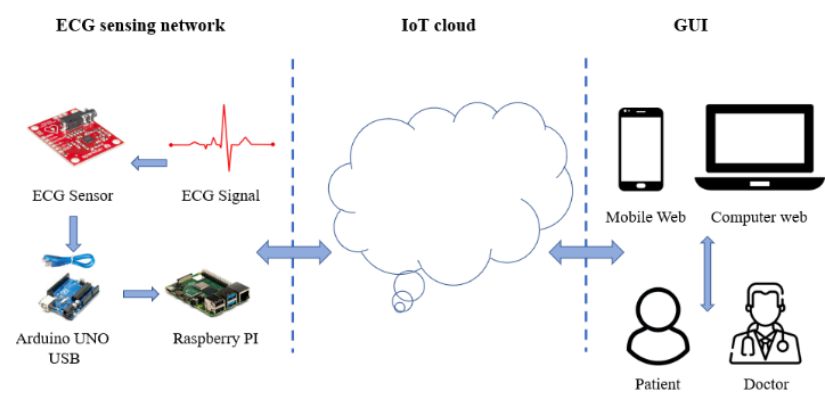

Figure 5. IoT-based ECG monitoring system 
Developing ECG measurement system is growing to achieve fully integrated system which reduce cost, power consumption, and size. Researchers proposed different architectures with different electrode types, data transmissions and performance accuracy that presented in Table 4 which provides a comparison among several ECG monitoring systems [89].

Table 4. Comparison among ECG Monitoring Systems

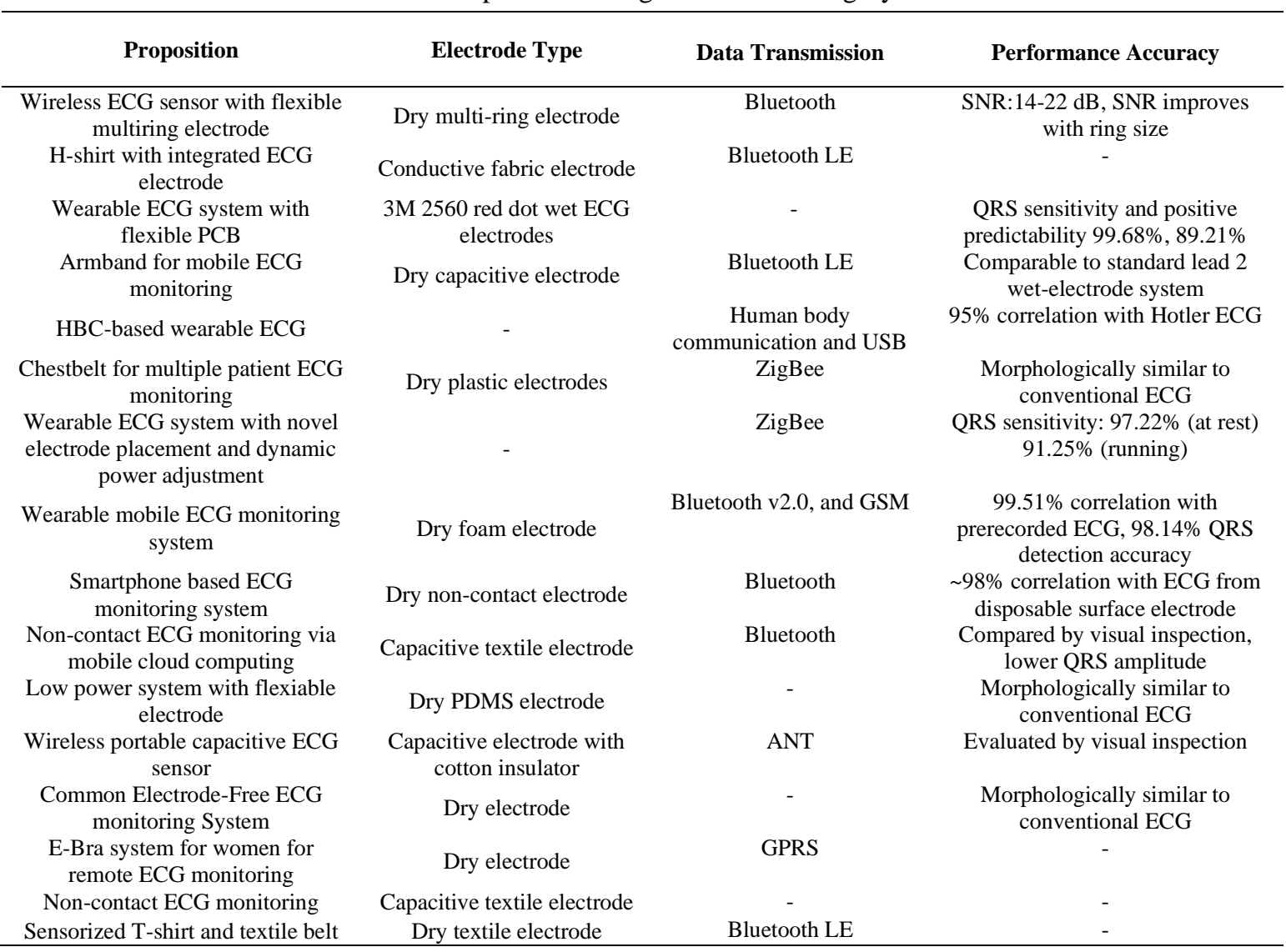

\subsection{ECG Sensing Network}

The ECG sensing network is considered as the core part of IoT-based ECG monitoring systems, as shown in Figure 5. The main objective for the ECG sensing network is to obtain ECG raw signal data from the user and then pass the data through a transmission medium such as Wi-Fi, Bluetooth, or Zigbee, as shown in Table 5, to the IoT cloud. The efforts of telecom companies, researchers, and institutes to deploy ECG sensors and monitorable wearable devices within the IoT environments has turned into a global trend, especially for those seeking to maintain a healthy lifestyle and obtain a reliable standard of security for their daily activities, actions, and interactions [41]. With ECG sensors, users can record their ECG signal data for hours or days. The recorded data must go through several preprocessing steps for the preservation of signal quality and to comply with wireless transmission standards. The most commonly used option to transmit these signals is Wi-Fi due to the limited ranges and capabilities of Bluetooth and Zigbee [88][41].

Table 5. Comparison of typical ECG sensing networks: Wi-Fi, Bluetooth, and Zigbee [41]

\begin{tabular}{|c|c|c|c|}
\hline $\begin{array}{l}\text { Standards / } \\
\text { Methods }\end{array}$ & $\begin{array}{l}\text { Wi-Fi-based ECG } \\
\text { Sensing Network }\end{array}$ & $\begin{array}{l}\text { Bluetooth-based ECG } \\
\text { Sensing Network }\end{array}$ & $\begin{array}{c}\text { ZigBee-based ECG } \\
\text { Sensing Network }\end{array}$ \\
\hline Protocol & IEEE 802.11 & IEEE 802.15.1 & IEEE 802.15.4 \\
\hline Coverage & $20-200 \mathrm{~m}$ & $20-30 \mathrm{~m}$ & $2-20 \mathrm{~m}$ \\
\hline Data Rates & 11-54 Mbps & 3-24 Mbps & $10-250 \mathrm{kbps}$ \\
\hline Power Consumption & Medium & Low & Low \\
\hline Terminal Dependency & $\begin{array}{l}\text { Data collection is } \\
\text { independent of smart } \\
\text { terminals }\end{array}$ & $\begin{array}{l}\text { Smart terminals are needed } \\
\text { for receiving and forwarding } \\
\text { sensed data }\end{array}$ & $\begin{array}{c}\text { Smart terminals are needed } \\
\text { for receiving and } \\
\text { forwarding sensed data }\end{array}$ \\
\hline
\end{tabular}




\subsection{IoT Cloud}

The purpose of the cloud in the IoT environments is to receive, store, process, analyze, and visualize the data. There are six basic layers that should exist in an IoT cloud to handle ECG signal data efficiently [90], as follows:

- Communication protocols

- Cloud server

- Data storage

- Data analysis

- Smart devices

- User application

\subsection{Graphical User Interface (GUI)}

The purpose of the GUI is to manage and provide the data to the end user via web virtualization method. End users can access their ECG data via web pages or applications with computing devices such as smartphones or laptops [90], [91].

\section{SIGNAL ACQUISITION APPROACHES}

Recently, ECG-based biometrics have progressed significantly toward commercial applications with the development of wearable technologies for ECG acquisition or the embedding of sensors into common objects. There are two main types of ECG biometric acquisition categories, namely on-the-person and off-theperson, as shown in Table 6.

Table 6. Comparison of on-the person and off-the-person acquisition

\begin{tabular}{ccc}
\hline Item & On-the-person & Off-the-person \\
\hline Type of Electrodes & Wet electrodes & Dry metallic electrodes \\
Number of Leads & 5,7, or 12 electrodes & 2 or 3 electrodes \\
Placement of Leads & Upper limbs (wrist, & Wrists, ankles, and chest \\
hands, or fingers) & No restriction \\
Movement & Limited & High \\
Noise & Low & Medium \\
Performance & High & \\
\hline
\end{tabular}

On-the-person acquisition uses the standard 12-lead configuration with 12 electrodes [92] or either the Frank lead configuration with seven electrodes [93] or Holter acquisition with five electrodes [94]. Based on medical standards and guidelines, acquisition devices use surface electrodes at specific points on the body surface with a conductive gel. Therefore, the on-the-person setup is unpractical for user authentication applications or the monitoring of daily activities since signal acquisition is performed with at least five electrodes. However, off-the-person ECG acquisition requires a smaller number of leads and has become more feasible for authentication systems based on ECG signals. Off-the-person ECG signals can be acquired from the upper limbs by using smartwatches and other wearable devices.

Kang et al. [95] proposed an authentication system design using ECG signals captured by an off-theperson wearable watch. They used the cross-correlation of templates extracted during the registration and authentication stages. Their results showed that the proposed system achieved a $5.2 \%$ false acceptance rate with $1.9 \%$ false rejection rate on average, with approximately 3 seconds needed for the authentication. ArteagaFalconi et al. [48] used an ECG sensor with two electrodes connecting to a smartphone for authentication purposes. The proposed algorithm requires at least 4 seconds for acquisition with reliable results as the true acceptance rate is around $82 \%$ while the false acceptance rate is approximately $1.4 \%$. Silva et al. [96] evaluated the permanence of ECG signals collected by using a sensor embedded into a keyboard wrist rest.

In order to apply IoT-cloud-based ECG authentication, many features need to be considered. These particularly include the number of electrodes, quality of ECG sensors, acquisition time, false acceptance rate, and true acceptance rate. The off-the-person approaches presented above are more suitable and realistic for IoT-cloud-based ECG authentication [50]. Yang et al. [45] and Wan et al. [97] proposed a new method for ECG monitoring based on the IoT cloud for medical purposes. The proposed architecture again consists of three parts, the ECG sensing network, IoT cloud, and GUI, as described in Figure 5.

\section{CHALLENGES AND OPPORTUNITIES OF WEARABLE AND SEAMLESSLY INTEGRATED DEVICES}

Wearable IoT devices contribute to the economy considerably from many perspectives and offer many benefits for people, such as providing continuous communication between devices and machines, continuous monitoring, high accuracy, low cost, elimination of repeated tasks, and user engagement. On the other hand, 
the increasing number and varieties of wearable devices has led to some security issues related to privacy and data protection [98].

According to the International Data Corporation's Worldwide Quarterly Wearable Device Tracker statement, 305.2 million units were shipped within the global market of wearable devices in 2019, a value that had grown by $71.4 \%$ from 178.0 million units in 2018 . The total volume is projected to grow to 489.1 million units in 2023; for example, approximately 69 million smartwatches were distributed in 2019, and it is estimated that 109.2 million units will be transported in 2023 [99]. These numbers show that the demand for wearable IoT devices will continue increasing sharply in the coming years. IoT systems have recently gained prominence due to the need for wearable devices in the healthcare industry [100].

The usage of wearable devices is increasing rapidly because they facilitate the interaction of users with other individuals and the surroundings. However, there are some challenges from the point of view of both the user and manufacturer that affect IoT systems, such as network communications, standardization, storage, scalability, flexibility, and power and energy efficiency [101]. One of the most important issues that wearable device manufacturers encounter is battery depletion. This issue of energy efficiency was addressed by Celic and Magjarevic [102], who proposed seamless connectivity between IoT devices and the network with low cost and power consumption. Their model architecture may deliver solutions for the usability of wearable devices.

Yildirim and Ali-Eldin [103] conducted a study proving that people using wearable IoT devices in the workplace have better motivation. This result indicates that technology may be adapted easily into our daily lives. Ramzan et al. [104] proposed a wearable apparel model to track drivers, communicate with mobile devices to generate warnings, and initiate any necessary precautionary operations while the user is driving a car. Most wearable device manufacturers have focused on specialized components for healthcare solutions. Moreover, companies need to redesign their production systems to adapt new service-based process models using IoT data to extend value chains and increase their revenues. Rymaszewska et al. [104] proposed a new framework enabling companies to produce valuations and use them in their server operations with IoT-built solutions.

As a result of the COVID-19 pandemic, many applications have recently been initiated to keep track of the movement of people and to ensure that safe physical distance is maintained between people as defined by the Centers for Disease Control and Prevention [106]. This distance can be monitored by Bluetooth IoT technologies that transmit data from one device to another in short ranges using Wi-Fi or other links. Recently, Google and Apple agreed to collaborate by sharing real-time user movement data to help governments and health organizations combat the COVID-19 pandemic. Vagus, an ECG smartwatch manufacturing company, has developed new ECG smartwatches to collect crowd-sourced data to measure COVID-19 contamination rates [107]. Fischer Connectors has also provided a solution for the easy integration of any IoT wearable application into a system with plug and use connectivity [108]. In other words, many companies are working on customizable and integrated solutions to improve communication performance and reliability.

\section{ECG DATABASES}

The Telemetric and Holter ECG Warehouse (THEW) [109] is one of the important resources for ECG databases. This initiative provides access to electrocardiographic data for the design of analytic methods to enlarge the pool of available information from datasets. These databases are accessible upon request. Open access ECG databases such as the MIT-BIH Arrhythmia Database, Creighton University Ventricular Tachycardia Arrhythmia Database, and European ST-T Database have led to the development and expansion of many techniques and methodologies. The American Heart Association (AHA) Database, which is not available publicly, also has 154 beat-by-beat annotated recordings of arrhythmias and normal ECG results. Moreover, the MIT PhysioNet initiative is one of the most important ECG repositories to be used for ECGrelated research. For example, one of the open access PhysioNet databases, PTB-XL ECG, contains a large set of datasets collected with devices from Schiller AG between October 1989 and June 1996, comprising data for 18885 patients from 12-lead ECGs of 10 seconds in length. Large quantities of electrocardiographic data should be tested to get higher accuracy for machine learning algorithms using ECG datasets.

CardioComm Solutions, Inc. offers a heart rhythm monitor device to measure the risk of atrial fibrillation by comparing ECG results with manual pulse monitoring using the THEW database. Alfaras et al. [110] presented a study that used the MIT-BIH Arrhythmia (MIT-BIH AR) and AHA databases. In this work, each database was divided into two sets of training and testing datasets to eliminate data duplication for different types of heartbeats, similar to a real environment. While 22 ECG records from MIT-BIT AR were used for training and testing, 79 ECG records were used for training and 75 records were used for testing the AHA Database. Another study [111] classified heartbeats using a DNN to evaluate a proposed model using two dissection structures of the MIT-BIH Arrhythmia Database. Table 7 represent a comparison between the most recognized datasets repositories for ECG data in term of purpose, access type and features. 
Table 7. Comparison among ECG datasets in term of purpose, access type and features

\begin{tabular}{llll}
\hline Datasets & \multicolumn{1}{c}{ Purpose } & Access Type & Data features \\
\hline $\begin{array}{l}\text { Telemetric and Holter ECG } \\
\text { Warehouse (THEW) }\end{array}$ & Design analytic methods & Upon Request & $\begin{array}{l}293 \text { GB, 3700 digital 24- } \\
\text { Holter ECG recordings }\end{array}$ \\
MIT-BIH arrhythmia database & Detection of heart arrhythmia & Open Access & 48 records, 30 minutes each \\
$\begin{array}{l}\text { Creighton University ventricular } \\
\text { tachycardia arrhythmia database }\end{array}$ & $\begin{array}{l}\text { Episodes of sustained ventricular } \\
\text { tachycardia, ventricular flutter, and } \\
\text { ventricular fibrillation }\end{array}$ & Open Access & 35 records, 8 minutes each \\
European ST-T database & Assessing the quality of ambulatory & Open Access & 90 records, two hours each \\
Emerican Heart & Association monitoring (AECG) systems \\
(AHA) database & $\begin{array}{l}\text { Evaluation of ventricular arrhythmia } \\
\text { detectors }\end{array}$ & Open Access & 80 records, 35 minutes each \\
The LTST Database & Detect coronary artery disease & Open Access & 24-hour ambulatory \\
records
\end{tabular}

Recently, Chapman University and Shaoxing People's Hospital (Shaoxing Hospital, Zhejiang University School of Medicine) initiated an ECG database for 12-lead ECG signals [112]. This repository has data for a total of 10646 patients' ECGs, including 5956 male and 4690 female patients. In addition to basic ECG measurements, this database contains QRS counts, atrial beat rates, ventricle beat rates, Q offsets, and T offsets. While $80 \%$ of the data was used to train the extreme gradient boosting tree classification model used for regression and classification problems in machine learning, $20 \%$ of the data was used to test this model.

The LTST Database contains 24-hour ambulatory registers selected from Holter recordings in Europe and the United States between 1994 and 2000 with clinical information in the Waveform Database (WFDB) format for waveform data, true QRS annotations, and ST annotations. This repository is suitable for IoT environments due to its use of the WFDB format, which can be applied with any devices [113].

Sadrawi et al. [114] evaluated arrhythmias with wearable ECG devices using 169 records from the American Heart Association Database (AHADB), Creighton University Ventricular Tachyarrhythmia Database (CUDB), MIT-BIH Arrhythmia Database (MITDB), and MIT-BIH Noise Stress Test Database (NSTDB). Moreover, a performance analysis of QRS detectors was performed by Liu et al. [115] based on four experiments using 516 records from different repositories such as the MITDB, MIT-BIH NSR, and Harvard Dataverse TELE Database.

\section{ECG SIGNAL PREPROCESSING AND FEATURE EXTRACTION \\ 9.1. ECG Signal Preprocessing}

ECG signal filtering: In order to maximize beat detection efficiency and eliminate baseline wander, obtained ECG signals must go through a filtering process. The high-pass filtering technique is widely used in the ECG filtering step to remove baseline wander by subtracting a low-frequency trend line from recorded ECG signals. Generally, by setting the window size between 500 and 750 points and applying a triangular (two-pass) moving average filter, the trend line of the ECG signal is formed. With the intention of eliminating high-frequency noise, a low-pass filter is used along with a triangular moving average filter and a window size of 3 points. Moving average filters provide a simple, valuable, and fast method for filtering ECG signals [116].

ECG signal segmentation: Applying a segmentation technique requires a certain duration of time to be set for all of the ECG segments. This time duration is most likely to be between 5 and 20 minutes for all segments, and it must be specified for analysis of the HRV. The first step in this technique is to pass moving windows over all recorded ECG data. Then, for each window in this time duration, statistical information on the ECG data is collected. In most related research work on ECG signals and feature extraction, a list of functional ECG segments is generated based on output statistics [117]. For the applicable segment based on the specified time duration, the selection criteria are as follows:

- Mean heart rate

- Overall number of ectopic beats

- No missing vital data

- Ocular observation of noise and ecotype 


\subsection{ECG Signal Feature Extraction}

QRS detection: In general, a correlation-based template matching algorithm is used to locate QRS complexes. However, to apply the template matching technique, there are two types of QRS templates that need to be used: global and self templates. As the first step before using the QRS templates, an averaging process must be performed for a specified number of QRS complexes in order to create the templates. A comparison of the created templates is achieved by computing a correlation coefficient, which is decided by the researchers based on several factors such as the nature of the ECG data, the aim of the study, or the targeted age of the subjects. When the global template fails to detect the QRS complex of the signal, the self template is used as a backup to perform the QRS detection process [118].

Inter-beat interval (IBI) extraction: Commonly, due to ECG signal physiology, the $\mathrm{R}$ wave is often a very straightforward wave to be recognized, and it typically has the largest amplitude in comparison with the surrounding $\mathrm{P}, \mathrm{Q}, \mathrm{S}$, and $\mathrm{T}$ waveforms. Therefore, researchers rely on the R wave to find the temporal locations of beats. The time difference between consecutive $\mathrm{R}$ peaks (or the $\mathrm{RR}$ interval) represents a beat-to-beat interval. Furthermore, NN (normal-to-normal) intervals are correlated with RR intervals, which originate from normal sinus rhythms [119], [120]. To calculate the time series of the IBI of an ECG segment containing $\mathrm{N}$ beats, the following equation is used:

$$
\operatorname{IBI}(n)=\operatorname{beat}(n+1)-\operatorname{beat}(n), 1 \leq n \leq N-1
$$

Here, $n$ is the time location of the $n^{\text {th }}$ beat.

Three consecutive ECG R peaks along with time and amplitude, as shown in Figure 6, represent the IBI between each pair of $\mathrm{R}$ peaks over a certain time interval [120], [121].

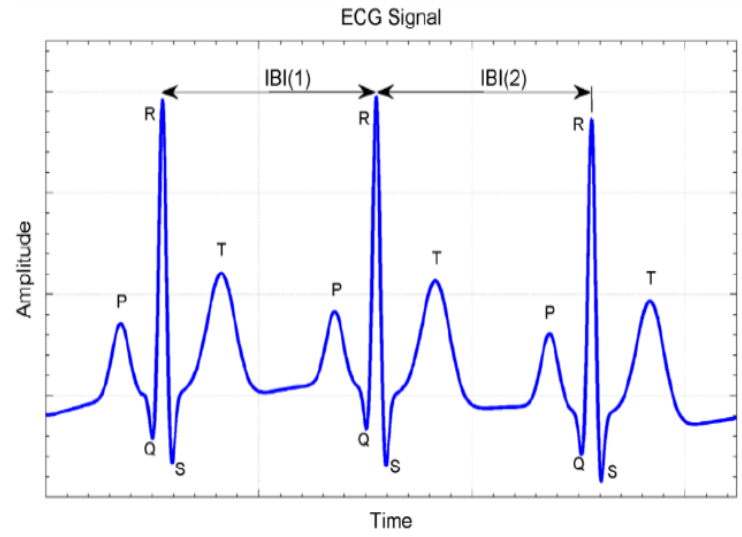

Figure 6. Determination of IBI based on R waves

The conventional methods commonly used by researchers for ECG preprocessing and feature extraction steps are listed in Table 8.

Table 8. Conventional methods for ECG preprocessing and feature extraction [118]

\begin{tabular}{cc}
\hline $\begin{array}{c}\text { QRS Detection } \\
\text { (Threshold) }\end{array}$ & Preprocessing (Filter Technique) \\
\hline QRS Amplitude & Low-pass filter and curve length transform \\
Root Mean Square (RMS) & Discrete wavelet transform (DWT) \\
Mean Deviation (MD) & DWT \\
Slope Criterion & Bandpass filter \\
Signal Peak and Noise & Bandpass filter, derivative, squaring, and \\
Peak & moving window integrator \\
Modulus Maxima & Continuous wavelet transform \\
\hline
\end{tabular}

\section{5G IOT ARCHITECTURE AND INFRASTRUCTURE}

\subsection{G Architecture}

$5 \mathrm{G}$ technology is expected to meet IoT requirements such as the demands for multiple device connectivity, high data rates, and low latency. Several IoT architectures have been proposed and reviewed from different perspectives within the field of 5G-related research. In the works of Al-Fuqaha et al. [122] and Mahmoud et al. [123], a three-layer IoT architecture comprising perception, network, and application layers was presented. It was considered as the basic architecture for an IoT platform. 5G IoT architecture can be divided into five layers that involve data acquisition, analysis, and the sharing of information between different 
types of network devices [11], [124]. The architecture of 5G IoT layers is demonstrated in Figure 7. In more detail, the five layers may be described as follows:

The sensor layer is the physical layer of the system that consists of sensors used in different IoT applications, such as E-healthcare sensors (ECG wearable devices), smart manufacturing sensors, or smart home sensors.

The gateway layer is the low-power wide area network (LPWAN) in 5G IoT architecture. This layer can be based on one of the following technologies: Wi-Fi, Sigfox, LoRa, ZigBee, or NB-IoT [125]. The Narrowband Internet of Things (NB-IoT) is a new wireless system introduced in 3GPP Release 13 that focuses on indoor coverage with a high number of connected devices.

The network layer is the backbone of IoT architecture as it transfers and exchanges information within a wide range. This layer includes a mobile network such as $5 \mathrm{G}$ or $4 \mathrm{G}$, a satellite communication network, and optical fiber communication.

The service layer is the IoT platform where cloud computing and big data analytics are implemented.

The $5 G$ IoT application layer is responsible for providing services to all applications, which may be smart homes, smart cities, E-healthcare, smart factories, or smart transportation, among others [11].

\begin{tabular}{|c|c|}
\hline Application Layer & $\begin{array}{l}\text { - E-Healthcare } \\
\text { - Smart Homes }\end{array}$ \\
\hline Service Layer & - Cloud Platform \\
\hline Network Layer & $\begin{array}{l}\text { - Mobile Network (5G, } 4 \mathrm{G}) \\
\text { - Satellite Communication }\end{array}$ \\
\hline Gateway Layer & $\begin{array}{l}\cdot \text { Wi-Fi } \\
\cdot \text { NB-IoT }\end{array}$ \\
\hline Sensor Layer & $\begin{array}{l}\text { - Medical Sensor (Wearable ECG } \\
\text { sensor, temperature sensor, etc.) }\end{array}$ \\
\hline
\end{tabular}

Figure 7. Architecture of 5G IoT

\subsection{G Infrastructure}

$5 \mathrm{G}$ technology is the main infrastructure for future health services and IoT services, particularly for ECG biometric authentication. 5G technology provides lower latency, higher capacity, and greater bandwidth, which are the main requirements for supporting ECG authentication. One of the main characteristics of 5G wireless networks is massive machine-type communication, which requires high connectivity density for IoT applications such as smart cities [126]. 5G technology will use the existing LTE frequency range (600 $\mathrm{MHz}$ to $6 \mathrm{GHz}$ ) with the new millimeter wave bands $(24-86 \mathrm{GHz})$. The new spectrum is expected to have high throughput, lower latency, and high connection density. A 5G wireless network composed of a new-generation radio access network (NG-RAN) and a 5 G core network (5GC) is shown in Figure 8.

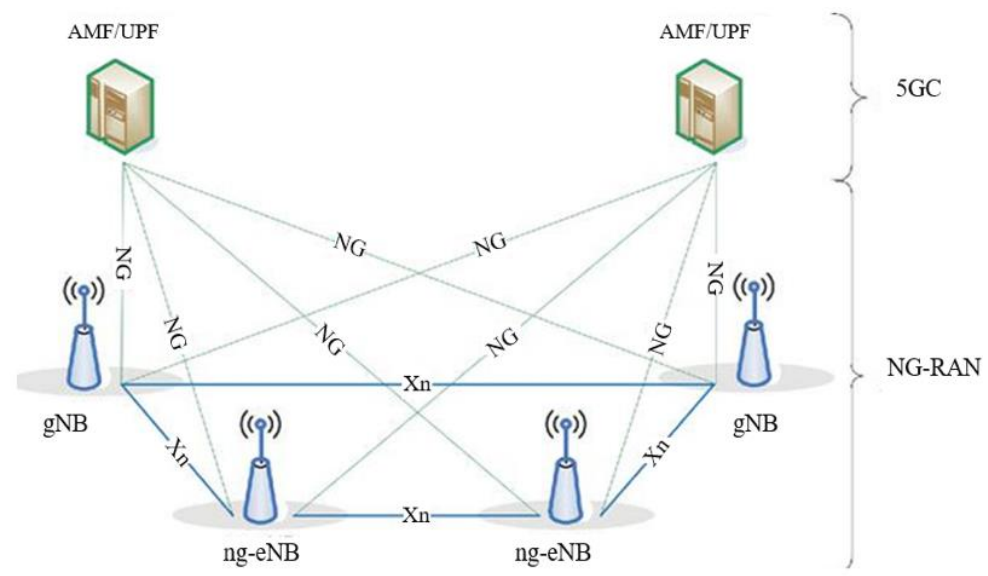

Figure 8. NG-RAN and 5G core system [127] 
The NG-RAN (ng-eNBs and gNBs) offers NR 5G or LTE radio for the core network. A base station can be gNB (a 5G base station), providing control and user plane services, or ng-eNB (New Generation Evolved Node B), providing LTE/E-UTRAN radio services. The Xn interface is a logical interface between two NG-RAN nodes that establishes a logical connection them, while NG interfaces connect the NG-RAN nodes to the AMF (Access and Mobility Management Function) and to the UPF (User Plane Function) in the 5GC [127]. The powerful characteristics of 5G technology as mentioned above will enable the use of ECG as a new promising biometric tool for authentication and identification in the future.

\section{ECG DEPLOYMENT OPPORTUNITIES AND CHALLENGES}

A rapid increase in the use of IoT devices and applications in most healthcare systems is currently being experienced in accordance with the increasing growth of the world's population and the accompanying increase in various chronic heart diseases. Thus, the IoT has a vital role to play in easing the diagnosis process of many cardiovascular diseases in addition to its use in advanced portable devices, making systems more effective and efficient. Future IoT systems should be able to handle many concerns for medical and biometric authentication systems, such as nano sensors sizes, long-term monitoring which is triggering big data issue, and these systems should be highly sensitive to ECG signals. Therefore, IoT systems must be able to handle both big data and nanoelectronics.

The electrical properties and conductivity of ECG sensors and devices will be improved with the incorporation of nanomaterials, which are also easily available, and this will reduce the costs. The sensors of IoT devices will allow ECG signals to be transmitted via their gateways using communication protocols such as 5G, LAN, Wi-Fi, and Bluetooth. These data can then be used by biometric authentication systems or can be sent to the cloud or healthcare systems for processing or analysis [128]. Therefore, the IoT faces another question of how it should assist the ECG acquisition framework to ensure secure data transmission [129], because the IoT generally transmits information through open channels.

As the numbers of IoT users and devices are increasing exponentially, huge amounts of data are being generated. Therefore, big data analytics is emerging as a very important field of technology that will help in improving decision-making, extracting useful information, and performing data analysis with the use of artificial intelligence algorithms and storage. For wearable and seamless IoT devices to achieve such improved systems with complete data collection, the number of electrodes has to be reduced, which is more challenging, and in this case, the signal quality must also be considered [130]. In addition, most of the current and future IoT devices that monitor ECG signals are shifting toward limb leads instead of chest leads, which affects the strength and quality of the signal and will have a negative impact due to the distance from the heart. Many studies have also shown that the use of these devices while exercising and the presence of movement artifacts, in addition to fatigue and different postures, will affect the recognition performance [131], [132].

The current trend in ECG signal acquisition is an evolution toward off-the-person acquisitions with an increasing focus on wearable and seamless devices, which leads to an increasing number of noise sources. Therefore, more intelligent filters and algorithms must be proposed, in addition to robust and adaptable denoising techniques. To achieve remarkable robustness to noise, many researchers have proposed the use of deep learning methodologies, which may result in better performance in signal denoising [133], [134]. The great potential of deep learning in ECG biometrics should be taken into consideration to provide optimized and coordinated solutions by integrating the four stages of denoising, preparation, feature extraction, and decisionmaking into one model, leading ECG authentication systems to new levels of robustness and overcoming the challenges of noise and variability. Furthermore, ECG biometric systems should be able to reduce computational costs and provide adequately powerful hardware, particularly with IoT devices, to perform accurately with short ECG segments, which will be a difficult goal to achieve [40].

Wearable devices today collect massive amounts of medical information using various health monitoring applications and such information needs to be placed behind a security wall to prevent it from being accessed and abused by adversaries. Thus, IoT security levels, protocols, and standards must be taken into consideration. Researchers need to look at the relationship between the mobility of users and the quality of data collected, as the duration of data collection in ECG biometric systems will affect the quality of the signals due to the low sensing capacity of wearable devices in addition to inevitable muscle activity [135]. Alongside the above challenges and future possibilities, there are also some psycho-physiological factors that challenge the use of ECG signals as a biometric trait in biometric authentication systems. These include short-term factors, such as the age and health condition of the user or geometric-related variations that can occur due to changes in position and orientation, and long-term factors such as mental and emotional conditions [136].

\section{CONCLUSION}

This survey has explored different perspectives and issues regarding the opportunities and challenges that have a direct impact on utilizing ECG signals in biometric authentication systems to be effectively designed 
and deployed in the 5G IoT environments. The design of ECG biometric systems varies based on a number of factors such as the type of device, the number of leads used, the time taken to collect data, and the portability and size of the device. IoT devices currently collect massive amounts of medical information using various health monitoring systems and applications. This personal information needs to be placed behind a security wall so that it cannot be accessed and abused by adversaries. Accordingly, IoT security levels, protocols, and standards must be carefully evaluated.

Some psycho-physiological factors also affect the usage of ECG signals as a biometric trait in biometric authentication systems, including short-term factors such as the age and health condition of the user, distorted signals due to geometric-related variations that can occur with changes in the position and orientation of the heart, and long-term factors such as mental and emotional conditions. These may cause high levels of noise that could obscure the signals, and so these factors must also be addressed. This survey has attempted to pave the way toward the effective and efficient implementation of ECG biometric authentication systems within the 5G IoT architecture and infrastructure by presenting essential knowledge regarding the deployment process of such systems.

This survey has also provided detailed information on the factors that influence the utilization of ECG signals in biometric authentication systems. Some of these factors are associated with signal morphology; some factors affect the ECG signals in addition to the deployment of ECG within the 5G IoT environments. The commonly used approaches for ECG signal acquisition have also been provided in detail here in the process of considering the challenges and opportunities of wearable and seamlessly integrated devices, as this is the current mainstream trend in the field of IoT research and industry. The ECG databases most commonly used to obtain raw signal data have been reviewed here, along with an explanation of the preprocessing and feature extraction of the signals, the 5G IoT architecture and infrastructure, and, finally, the opportunities and challenges of ECG deployment.

In future work, we plan to pursue the implementation of standard biometric authentication systems. Targeted standard systems must be characterized as effective active systems that are efficiently compatible with 5G IoT architecture and infrastructure.

\section{REFERENCES}

[1] The Internet of things (IoT): Applications, Technology, And Privacy Issues, Nova Publisher, New York, NY 2016.

[2] D. Mishra, A. Gunasekaran, S. J. Childe, T. Papadopoulos, R. Dubey, and S. Wamba, "Vision, applications and future challenges of Internet of Things: A bibliometric study of the recent literature," Ind. Manag. Data Syst., vol. 116, no. 7, pp. 1331-1355, Aug. 2016.

[3] Amazon IoT Core. 2020. [Online]. Available: https://aws.amazon.com/iot-core

[4] What is Azure Internet of Things (IoT)? [Online]. Available: https://docs.microsoft.com/en-us/azure/iotfundamentals/iot-introduction

[5] Google Cloud IoT Core overview. 2020. [Online]. Available: https://cloud.google.com/iot/docs/concepts/ overview

[6] H. S. Maghdid, K. Z. Ghafoor, A. S. Sadiq, K. Curran, and K. Rabie, "A novel ai-enabled framework to diagnose coronavirus COVID 19 using smartphone embedded sensors: design study,” ArXiv, ArXiv200307434 Cs Q-Bio, Mar. 2020.

[7] G. Lampropoulos, K. Siakas, and T. Anastasiadis, "Internet of things in the context of industry 4.0: An overview," Int. J. Entrep. Knowl., vol. 7, no. 1, pp. 4-19, Jun. 2019.

[8] The Impact of COVID-19 on Sustainability and Technology. 2020. [Online]. Available: https://blogidcuk.com/impact-covid-19-sustainability-technology/

[9] F. Samie, L. Bauer, and J. Henkel, "From cloud down to things: An overview of machine learning in internet of things,” IEEE Internet Things J., vol. 6, no. 3, pp. 4921-4934, Jun. 2019.

[10] Number of smartphone users worldwide from 2016 to 2021. 2020. [Online]. Available: https://www.statista.com/statistics/330695/number-of-smartphone-users-worldwide/

[11] L. Chettri and R. Bera, "A comprehensive survey on internet of things (IoT) toward 5G wireless systems," IEEE Internet of Things Journal, vol. 7, no. 1, pp. 16-32, Jan. 2020.

[12] E. Kapassa, M. Touloupou, P. Stavrianos and D. Kyriazis, "Dynamic 5G Slices for IoT Applications with Diverse Requirements," in Proc. Fifth International Conference on Internet of Things: Systems, Management and Security, Valencia, 2018, pp. 195-199.

[13] M. M. Alsulami and N. Akkari, "The role of 5G wireless networks in the internet-of- things (IoT)," in Proc. 1st International Conference on Computer Applications \& Information Security (ICCAIS), Riyadh, 2018, pp. 1-8.

[14] A. K. Jain, R. Acharya, S. Jakhar and T. Mishra, "Fifth Generation (5G) Wireless Technology "Revolution in Telecommunication," in Proc. Second International Conference on Inventive Communication and Computational Technologies (ICICCT), Coimbatore, 2018, pp. 1867-1872.

[15] E. Balevi and R. D. Gitlin, “A Random Access Scheme for Large Scale 5G/IoT Applications,” in Proc. 2018 IEEE 5G World Forum (5GWF), Silicon Valley, CA, 2018, pp. 452-456. 
[16] S. Li, Y. Liu, L. Lin, X. Sun, S. Yang and D. Sun, "Millimeter-Wave Channel Simulation and Statistical Channel Model in the Cross-Corridor Environment at 28 GHz for 5G Wireless System," in Proc. 2018 International Conference on Microwave and Millimeter Wave Technology (ICMMT), Chengdu, 2018, pp. 1-3.

[17] A. H. Mohsin, A. A. Zaidan, B. B. Zaidan, O. S. Albahri, S. A. Ariffin, A. Alemran, O. Enaizan, A. H. Shareef, A. N. Jasim, N. S. Jalood, M. J. Baqer, "Finger Vein Biometrics: Taxonomy Analysis, Open Challenges, Future Directions, and Recommended Solution for Decentralised Network Architectures," IEEE Access, vol. 8, pp. 9821-9845, 2020.

[18] R. Tolosana, M. Gomez-Barrero, C. Busch and J. Ortega-Garcia, "Biometric Presentation Attack Detection: Beyond the Visible Spectrum," IEEE Transactions on Information Forensics and Security, vol. 15, pp. 1261-1275, 2020.

[19] V. M. Patel, R. Chellappa, D. Chandra and B. Barbello, "Continuous User Authentication on Mobile Devices: Recent progress and remaining challenges,” IEEE Signal Processing Magazine, vol. 33, no. 4, pp. 49-61, July 2016.

[20] H. Feng, K. Fawaz, K. G. Shin, Continuous Authentication for Voice Assistants. in Proc. MobiCom, Snowbird, UT, 2017, pp. 343-355.

[21] A. Das, O. K. Manyam, M. Tapaswi, and V. Taranalli. "Multilingual spoken-password based user authentication in emerging economies using cellular phone networks," in Proc. 2008 IEEE Workshop on Spoken Language Technology SLT 2008,". Goa, Indiana, 2008, pp. 5-8.

[22] M. Baloul, E. Cherrier, and C. Rosenberger. "Challenge based speaker recognition for mobile authentication," in Proc. 2012 BIOSIG International Conference of Biometrics Special Interest Group (BIOSIG)" - Darmstadt, Germany, 2012, pp. $1-7$.

[23] W. J. Herschel. (December 1916). The origin of finger-printing Nature, vol. 98, no. 2458, p. 268, 1916. [Online]. Available: https://doi.org/10.1038/098268a0.

[24] J. Preciozzi, G. Garella, V. Camacho, F. Franzoni, L. Di Martino, G. Carbajal, and A. Fernandez, "Fingerprint Biometrics From Newborn to Adult: A Study From a National Identity Database System," IEEE Transactions on Biometrics, Behavior, and Identity Science, vol. 2, no. 1, pp. 68-79, Jan. 2020.

[25] T. Jaikla, S. Pichetjamroen, C. Vorakulpipat and A. Pichetjamroen, "A Secure Four-factor Attendance System for Smartphone Device," in Proc. 22nd International Conference on Advanced Communication Technology (ICACT), Phoenix Park, PyeongChang, Korea (South), 2020, pp. 65-68.

[26] S. Srihi, A. Balti, F. Fnaiech and H. Hamam, "Banking Security System Based on SVD Fingerprints and Cryptography Passwords," 2018 International Conference on Control, Automation and Diagnosis (ICCAD), Marrakech, Morocco, 2018, pp. 1-5, doi: 10.1109/CADIAG.2018.8751249

[27] E. G. Spanakis, M. Spanakis, A. Karantanas, and K. Marias. "Secure access to patient's health records using SpeechXRays a mutli-channel biometrics platform for user authentication," in Proc. 2016 38th Annual International Conference of the IEEE Engineering in Medicine and Biology Society (EMBC), Orlando, FL, 2016, pp. 2541-2544 .

[28] S. Das and J. Debbarma. "Designing a Biometric Strategy (Fingerprint) Measure for Enhancing ATM Security in Indian E-Banking System," International Journal of Information and Communication Technology Research, vol. 1, no. 5, Sep.2011.

[29] S. D. Shirke and C. Rajabhushnam, "Iris Recognition Using Visible Wavelength Light Source and Near Infrared Light Source Image Database: A Short Survey," in Proc. 2019 3rd International Conference on Trends in Electronics and Informatics (ICOEI), Tirunelveli, India, 2019, pp. 566-571.

[30] S. Patil, S. Gudasalamani and N. C. Iyer, "A survey on Iris recognition system," in Proc. 2016 International Conference on Electrical, Electronics, and Optimization Techniques (ICEEOT), Chennai, 2016, pp. 2207-2210.

[31] Klontz J., Burge M.J. (2016) Periocular Recognition from Low-Quality Iris Images. In: Bowyer K., Burge M. (eds) Handbook of Iris Recognition. Advances in Computer Vision and Pattern Recognition. Springer, London. https://doi.org/10.1007/978-1-4471-6784-6_14.

[32] G. M. Zafaruddin and H. S. Fadewar, "Face recognition: A holistic approach review," in Proc. 2014 International Conference on Contemporary Computing and Informatics (IC3I), Mysore, 2014, pp. 175-178.

[33] I. Masi, Y. Wu, T. Hassner and P. Natarajan, "Deep Face Recognition: A Survey," in Proc. 2018 31st SIBGRAPI Conference on Graphics, Patterns and Images (SIBGRAPI), Parana, 2018, pp. 471-478.

[34] M. U. Khan, S. Aziz, K. Iqtidar, A. Saud and Z. Azhar, "Biometric Authentication System Based on Electrocardiogram (ECG)," in Proc. 2019 13th International Conference on Mathematics, Actuarial Science, Computer Science and Statistics (MACS), Karachi, Pakistan, 2019, pp. 1-6.

[35] Y. Chen, C. Chen, C. Lin and C. Chiang, "Application of ECG Authentication in IoT-Based Systems," in Proc. 2018 International Conference on System Science and Engineering (ICSSE), New Taipei, 2018, pp. 1-6.

[36] R. German and K. S. Barber, "Current Biometric Adoption and Trends," UT CID Report \#18-02, The University of Texas at Austin, September 2017.

[37] Zion Market Research, "Biometric Technology Market By Application (Face Recognition, Fingerprint Recognition, Voice Recognition, Signature Recognition, Iris Recognition, Middleware Recognition, AFIS, Hand Geometry Recognition Non-AFIS, and Others) and By End-Use (Government, Banking \& Finance, Consumer Electronics, Healthcare, IT \& Telecommunication, Transport/Logistics, Defense \& Security, and Others): Global Industry Perspective, Comprehensive Analysis, and Forecast, 2018-2025".

[38] P. Corcoran and C. Costache, "Biometric Technology and Smartphones: A consideration of the practicalities of a 
broad adoption of biometrics and the likely impacts," in IEEE Consumer Electronics Magazine, vol. 5, no. 2, pp. 7078, April 2016.

[39] A. Das, C. Galdi, H. Han, R. Ramachandra, J. Dugelay and A. Dantcheva, "Recent Advances in Biometric Technology for Mobile Devices," in Proc. 2018 IEEE 9th International Conference on Biometrics Theory, Applications and Systems (BTAS), Redondo Beach, CA, USA, 2018, pp. 1-11.

[40] J. Ribeiro Pinto, J. S. Cardoso and A. Lourenço, "Evolution, Current Challenges, and Future Possibilities in ECG Biometrics," IEEE Access, vol. 6, pp. 34746-34776, 2018.

[41] P. Kamble and A. Birajdar, "IoT Based Portable ECG Monitoring Device for Smart Healthcare," in Proc. 2019 Fifth International Conference on Science Technology Engineering and Mathematics (ICONSTEM), Chennai, India, 2019, pp. 471-474.

[42] H. A. El Zouka and M. M. Hosni, "Secure IoT communications for smart healthcare monitoring system," Internet of Things, Jan. 2019.

[43] A. R. Moosavi, E. Nigussie, M. Levorato, S. Virtanen and J. Isoaho, "Performance Analysis of End-to-End Security Schemes in Healthcare IoT," Procedia Computer Science, vol. 130, 2018, pp. 432-439.

[44] N. M. M. AbdElnapi, N. F. Omran, A. A. Ali and F. A. Omara, "A survey of internet of things technologies and projects for healthcare services," in Proc. 2018 International Conference on Innovative Trends in Computer Engineering (ITCE), Aswan, 2018, pp. 48-55.

[45] Z. Yang, Q. Zhou, L. Lei, K. Zheng, and W. Xiang, "An IoT-cloud Based Wearable ECG Monitoring System for Smart Healthcare," J. Med. Syst., vol. 40, no. 12, p. 286, Dec. 2016.

[46] N. Shakhakarmi "5G Wireless Communications Systems: Heterogeneous Network Architecture and Design for Small Cells, D2D Communications (Low Range, Multi-hop) and Wearable Healthcare System on chip (ECG, EEG) for 5G Wireless," Int. J. Comput. Sci. Issues, vol. 13, no. 6, pp. 34-45, Nov. 2016.

[47] S. R. Islam, D. Kwak, M. H. Kabir, M. Hossain, and K.S. Kwak, "The Internet of Things for Health Care: A Comprehensive Survey," IEEE Access, vol. 3, pp. 678-708, 2015.

[48] J. S. Arteaga-Falconi, H. Al Osman and A. El Saddik, "ECG Authentication for Mobile Devices," IEEE Transactions on Instrumentation and Measurement, vol. 65, no. 3, pp. 591-600, March 2016.

[49] H. Tan and I. Chung, "Secure Authentication and Group Key Distribution Scheme for WBANs Based on Smartphone ECG Sensor,” IEEE Access, vol. 7, pp. 151459-151474, 2019.

[50] F. Samie, L. Bauer and J. Henkel, "IoT technologies for embedded computing: A survey," in Proc. 2016 International Conference on Hardware/Software Codesign and System Synthesis (CODES+ISSS), Pittsburgh, PA, 2016, pp. 1-10.

[51] S. Maqbool, M. Waseem Iqbal, M. Raza Naqvi, K. Sarmad Arif, M. Ahmed and M. Arif, "IoT Based Remote Patient Monitoring System," 2020 International Conference on Decision Aid Sciences and Application (DASA), 2020, pp. 1255-1260, doi: 10.1109/DASA51403.2020.9317213.

[52] G. Wang, D. John and A. Nag, "Low Complexity ECG Biometric Authentication for IoT Edge Devices," 2020 IEEE International Conference on Integrated Circuits, Technologies and Applications (ICTA), 2020, pp. 145-146, doi: 10.1109/ICTA50426.2020.9332012.

[53] C. Liu, X. Zhang, L. Zhao, F. Liu, X. Chen, Y. Yao, and J. Li, "Signal Quality Assessment and Lightweight QRS Detection for Wearable ECG SmartVest System," IEEE Internet of Things Journal, 2019, vol. 6, no. 2, pp. 1363-1374, doi: 10.1109/JIOT.2018.2844090.

[54] L. Xiaolin, B. Cardiff and D. John, "A 1D Convolutional Neural Network for Heartbeat Classification from Single Lead ECG," 27th IEEE International Conference on Electronics, Circuits and Systems (ICECS), 2020, pp. 1-2, doi: 10.1109/ICECS49266.2020.9294838

[55] A. Malik and R. K. Sharma, "Detection of heart conditions using HRV processor in Matlab simulink," in Proc. 2017 International Conference on Trends in Electronics and Informatics (ICEI), Tirunelveli, 2017, pp. 861-864.

[56] Y. Hagiwara, H. Fujita, S. Lih Oh, J. Hong Tan, R. San Tan, E. J Ciaccio, and U. Rajendra Acharya, "Computer-aided diagnosis of atrial fibrillation based on ECG Signals: A review," Information Sciences, vol. 467, ISSN 0020-0255, 2018. pp. 99-114.

[57] A. Y. Shdefat, M. Il Joo, and H. C. Kim, "A Method of Analyzing ECG to Diagnose Heart Abnormality utilizing SVM and DWT," Journal of Multimedia Information System, vol. 3, no. 2, pp. 35-42, Aug. 2016.

[58] S. Yazdani, P. Laub, A. Luca and J. Vesin, "Heart rhythm classification using short-term ECG atrial and ventricular activity analysis," in Proc. 2017 Computing in Cardiology (CinC), Rennes, France, 2017, pp. 1-4.

[59] A. Srinivasulu and N. Sriraam, "Ventricular Ectopic Beat Classification Using KNN Multi-Feature Classifier," in Proc. 2018 International Conference on Communication, Computing and Internet of Things (IC3IoT), Chennai, India, 2018, pp. 69-72.

[60] A. L. Fred, K. S. N, V. Suresh, R. A. Mathew, R. Reji and S. S. Mathews, "Hardware Implementation of Heart Rate and QRS Complex Detection Using Raspberry Pi Processor for Medical Diagnosis," in Proc. 2019 International Conference on Recent Advances in Energy-efficient Computing and Communication (ICRAECC), Nagercoil, India, 2019, pp. 1-4.

[61] X. Tang, Q. Hu and W. Tang, "A Real-Time QRS Detection System With PR/RT Interval and ST Segment Measurements for Wearable ECG Sensors Using Parallel Delta Modulators," IEEE Transactions on Biomedical Circuits and Systems, vol. 12, no. 4, pp. 751-761, Aug. 2018. 
[62] A. Appathurai, J. J. Carol, C. Raja, S.N. Kumar, A. V. Daniel, A. J. G. Malar, A. L. Fred, and S. Krishnamoorthy, "A study on ECG signal characterization and practical implementation of some ECG characterization techniques," Measurement, vol. 147, 2019.

[63] A. Y. Shdefat, M. Il Joo, S. H. Choi, and H. C. Kim, "Utilizing ECG Waveform Features as New Biometric Authentication Method," International Journal of Electrical and Computer Engineering (IJECE), vol. 8, no. 2, pp. 658665, April. 2018.

[64] D. A. B. Moreira et al., "Electrocardiogram Fiducial Points Detection for Health Care Systems," in Proc. 2018 IEEE Symposium on Computers and Communications (ISCC), Natal, 2018, pp. 00640-00645.

[65] D. Kumari, I. Saini and N. Sood, "Detection of Heart rate through Speech Using Mel Frequency cepstrum coefficients," in Proc. 2019 3rd International Conference on Trends in Electronics and Informatics (ICOEI), Tirunelveli, India, 2019, pp. 455-458.

[66] O. Divyabharathi, A. Sundar, C. Mukherjee and S. Deepika, "Analysis of Human Physiological Parameters Using Real-Time HRV Estimation from Acquired ECG Signals," in Proc. 2019 International Conference on Data Science and Communication (IconDSC), Bangalore, India, 2019, pp. 1-4.

[67] H. D. Hesar and M. Mohebbi, “An Adaptive Kalman Filter Bank for ECG Denoising,” IEEE Journal of Biomedical and Health Informatics, 2020.

[68] P. Kumar and V. K. Sharma, "Detection and classification of ECG noises using decomposition on mixed codebook for quality analysis," Healthcare Technology Letters, vol. 7, no. 1, pp. 18-24, 22020.

[69] Z. Gao, J. Wu, J. Zhou, W. Jiang and L. Feng, "Design of ECG Signal Acquisition and Processing System," in Proc. 2012 International Conference on Biomedical Engineering and Biotechnology, Macao, 2012, pp. 762-764.

[70] S. Abbaspour, A. Fallah, M. Linden, and H. Gholamhossein, "Filter bank-based processing of the stress ECG," in Proc. IEEE 39th Annu. Int. Conf. Eng. Med. Biol. Soc., Sep. 1995, pp. 887-888

[71] T. Y. M. Slonim, M. A. Slonim, and E. A. Ovsyscher, "The use of simple FIR Filters for filtering of ECG Signals and a New Method for Post-Filter Signal Reconstruction," in Proc. Comptr. Cardio. Conf., Sep. 1993, pp. 871-873.

[72] N.V. Thakor and Y.S. Zhu, "Applications of adaptive filtering to ECG analysis: Noise cancellation and arrhythmia detection,” IEEE Trans. Biomed. Eng., vol. 38, no. 8, pp. 785-794, Aug. 1991

[73] J. A. Sukor, "Signal quality measures for pulse oximetry and blood pressure signals acquired in unsupervised home telecare environments". Ph.D. dissertation, Uni. of New South Wales, 2012.

[74] C. Orphanidou, T. Bonnici, P. Charlton, D. Clifton, D. Vallance, L. Tarassenko, "Signal quality indices for the electrocardiogram and photoplethysmogram: derivation and applications to wireless monitoring," IEEE J. Biomed. Health Inf., vol. 19, no. 3, pp. 832-838, 2014.

[75] O. Sayadi and M. Shamsollahi, "A model-based Bayesian framework for ECG beat segmentation," Physiological Measurement, vol. 30, no. 3, pp. 335-352, 2009.

[76] P. E. McSharry, G. D. Clifford, L. Tarassenko, and L. A. Smith, "A dynamical model for generating synthetic electrocardiogram signals," IEEE Trans. Biomedical Engineering, vol. 50, no. 3, pp. 289-294, 2003.

[77] K. Dragomiretskiy, and D. Zosso, "Variational mode decomposition," IEEE Trans. Signal Process., vol. 62, no. 3, pp. 531-544, Feb. 2014.

[78] R. Sameni, M. B. Shamsollahi, C. Jutten, and G.D. Clifford, "A nonlinear Bayesian filtering framework for ECG denoising,” IEEE Trans. Biomed. Eng., vol. 54, no. 12, pp. 2172-2185, Dec. 2007.

[79] S. Jain, V. Bajaj, and A. Kumar, "Riemann Liouvelle Fractional Integral based Empirical Mode Decomposition for ECG Denoising,” IEEE J. Biomed. Health Informat., vol. 22, no. 4, pp. 1133-1139, Sept. 2017.

[80] M.A. Kabir, and C. Shahnaz, "Denoising of ECG signals based on noise reduction algorithms in EMD and wavelet domains," Biomed. Signal Process. Control, vol. 7, no. 5, pp. 481-489, Sept. 2012.

[81] A.O. Boudraa and J.C. Cexus, "EMD-based signal filtering,” IEEE Trans. Instrum. Meas., vol. 56, no. 6, pp. 21962202, Dec. 2007.

[82] S. Abbaspour, A. Fallah, M. Linden, and H. Gholamhossein, "Evaluation of wavelet based methods in removing motion artifact from ECG signal," in Proc. 16th Nordic-Baltic Conf. Biomed. Eng., Gothenburg, Sweden, Oct. 2015, pp. $1-4$.

[83] B.H. Tracey, and E.L. Miller, "Nonlocal means denoising of ECG signals," IEEE Trans. Biomed. Eng., vol. 59, no. 9, pp. 2383-2386, Sept. 2012.

[84] P. Singh, and G. Pradhan, "Exploring the Non-Local Similarity Present in Variational Mode Functions for Effective ECG Denoising,” in Proc. IEEE Int. Conf. Acoust., Speech Signal Process, Apr. 2018, pp. 861-865.

[85] P. Singh, S. Shahnawazuddin, and G. Pradhan, "Significance of modified empirical mode decomposition for ECG denoising," in Proc. IEEE 39th Annu. Int. Conf. Eng. Med. Biol. Soc., July 2017, pp. 2956-2959.

[86] P. Singh and G. Pradhan, "A New ECG Denoising Framework Using Generative Adversarial Network," in IEEE/ACM Transactions on Computational Biology and Bioinformatics.

[87] T. Shaown, I. Hasan, M. M. R. Mim and M. S. Hossain, "IoT-based Portable ECG Monitoring System for Smart Healthcare," in Proc. 2019 1st International Conference on Advances in Science, Engineering and Robotics Technology (ICASERT), Dhaka, Bangladesh, 2019, pp. 1-5. 
[88] M. A. G. Santos, R. Munoz, R. Olivares, P. P. R. Filho, J. Del Ser., and V. H. C. de Albuquerque, "Online heart monitoring systems on the internet of health things environments: A survey, a reference model and an outlook," Information Fusion, vol. 53, 2020, pp. 222-239.

[89] S. Majumder, L. Chen, O. Marinov, C. Chen, T. Mondal and M. J. Deen, "Noncontact Wearable Wireless ECG Systems for Long-Term Monitoring," in IEEE Reviews in Biomedical Engineering, vol. 11, pp. 306-321, 2018, doi: 10.1109/RBME.2018.2840336.

[90] L. M. Vela, H. Kwon, S. B. Rutkove and B. Sanchez, "Standalone IoT Bioimpedance Device Supporting Real-Time Online Data Access," IEEE Internet of Things Journal, vol. 6, no. 6, pp. 9545-9554, Dec. 2019.

[91] P. Singh and A. Jasuja, "IoT based low-cost distant patient ECG monitoring system," in Proc. 2017 International Conference on Computing, Communication and Automation (ICCCA), Greater Noida, 2017, pp. 1330-1334.

[92] R. D. Labati, R. Sassi and F. Scotti, "ECG biometric recognition: Permanence analysis of QRS signals for 24 hours continuous authentication," in Proc. 2013 IEEE International Workshop on Information Forensics and Security (WIFS), Guangzhou, 2013, pp. 31-36.

[93] L. Biel, O. Pettersson, L. Philipson and P. Wide, "ECG analysis: a new approach in human identification," IEEE Transactions on Instrumentation and Measurement, vol. 50, no. 3, pp. 808-812, June 2001.

[94] G. Wübbeler, M. Stavridis, D. Kreiseler and R. Bousseljot, C. Elster, "Verification of humans using the electrocardiogram," Pattern Recognition Letters, vol. 28, no.10, pp.1172-1175, July 2007

[95] S. J. Kang, S. Y. Lee, H. I. Cho and H. Park, "ECG Authentication System Design Based on Signal Analysis in Mobile and Wearable Devices," IEEE Signal Processing Letters, vol. 23, no. 6, pp. 805-808, June 2016.

[96] H. Plácido da Silva, A. Fred, A. Lourenco and A. Jain, "Finger ECG signal for user authentication: Usability and performance," in Proc. 2013 IEEE 6th International Conference on Biometrics: Theory, Applications and Systems (BTAS), Arlington, VA, 2013, pp. 1-8.

[97] J. Wan, M. Al-awlaqi, M. Li, M. O'Grady, X. Gu, J. Wang and N. Cao, "Wearable IoT enabled real-time health monitoring system," EURASIP Journal on Wireless Communications and Networking, no. 1, pp. 298, 2018.

[98] V. E. Balas, V. K. Solanki, R. Kumar, and Md. A. R. Ahad, A handbook of internet of things in biomedical and cyber physical system, 2020 .

[99] IDC Worldwide Quarterly Wearable Device Tracker. [Online]. Available: https://www.idc.com/getdoc.jsp?containerId=prUS45737919.

[100] T. Poongodi, R. Krishnamurthi, R. Indrakumari, P. Suresh, and B. Balusamy, "Wearable Devices and IoT," A Handbook of Internet of Things in Biomedical and Cyber Physical System, Springer, Cham, 2020, pp. 245-273.

[101] Darwish, A. E. Hassanien, M. Elhoseny, A. K. Sangaiah, and K. Muhammad, "The impact of the hybrid platform of internet of things and cloud computing on healthcare systems: opportunities, challenges, and open problems," J. Ambient Intell. Humaniz. Comput., vol. 10, no. 10, pp. 4151-4166, Oct. 2019.

[102] L. Celic and R. Magjarevic, "Seamless connectivity architecture and methods for IoT and wearable devices," Automatika, vol. 61, no. 1, pp. 21-34, Jan. 2020.

[103] H. Yildirim and A. M. T. Ali-Eldin, "A model for predicting user intention to use wearable IoT devices at the workplace,” J. King Saud Univ. - Comput. Inf. Sci., vol. 31, no. 4, pp. 497-505, Oct. 2019.

[104] M. Ramzan, S. M. Awan, H. Aldabbas, A. Abid, M. Farhan, S. Khalid, and R. M. A. Latif, "Internet of medical things for smart D3S to enable road safety,” Int. J. Distrib. Sens. Netw., vol. 15, no. 8, Aug. 2019.

[105] Rymaszewska, P. Helo, and A. Gunasekaran, "IoT powered servitization of manufacturing - an exploratory case study,” Int. J. Prod. Econ., vol. 192, pp. 92-105, Oct. 2017.

[106] Social Distancing, Quarantine, and Isolation. [Online]. Available: https://www.cdc.gov/coronavirus/2019ncov/prevent-getting-sick/social-distancing.html

[107] Coronavirus COVID-19 crowd sourced open data study. [Online]. Available: https://vagus.co/covid-19-study/

[108] IoT \& WEARABLE connectors and assembled solutions. [Online]. Available: https://www.fischerconnectors.com/global/en/applications/internet-things-iot-wearables

[109] J. Couderc, "The telemetric and holter ECG warehouse initiative (THEW): A data repository for the design, implementation and validation of ECG-related technologies," in Proc. 2010 Annual International Conference of the IEEE Engineering in Medicine and Biology, Buenos Aires, Aug. 2010, pp. 6252-6255.

[110] M. Alfaras, M. C. Soriano, and S. Ortín, "A Fast Machine Learning Model for ECG-Based Heartbeat Classification and Arrhythmia Detection,” Front. Phys., vol. 7, p. 103, Jul. 2019.

[111] H. Shi, C. Qin, D. Xiao, L. Zhao, and C. Liu, "Automated heartbeat classification based on deep neural network with multiple input layers,” Knowl.-Based Syst., vol. 188, p. 105036, Jan. 2020.

[112] J. Zheng, J. Zhang, S. Danioko, H. Yao, H. Guo, and C. Rakovski, "A 12-lead electrocardiogram database for arrhythmia research covering more than 10,000 patients," Sci. Data, vol. 7, no. 1, p. 48, Dec. 2020.

[113] F. Jager, A. Taddei, G. B. Moody, M. Emdin, G. Antolič, R. Dorn, A. Smrdel, C. Marchesi, R. G. Mark, "Long-term ST database: a reference for the development and evaluation of automated ischaemia detectors and for the study of the dynamics of myocardial ischaemia," Med. Biol. Eng. Comput., vol. 41, no. 2, pp. 172-182, Mar. 2003.

[114] M. Sadrawi, C. H. Lin, Y. T. Lin, Y. Hsieh, C. C. Kuo, J. C. Chien, K. Haraikawa, M. F. Abbod, J. S. Shieh, "Arrhythmia Evaluation in Wearable ECG Devices," Sensors, vol. 17, no. 11, p. 2445, Oct. 2017 
[115] F. Liu, C. Liu, X. Jiang, Z. Zhang, Y. Zhang, J. Li, and S. Wei, "Performance Analysis of Ten Common QRS Detectors on Different ECG Application Cases," J. Healthc. Eng., pp. 9050812-9050812, 2018.

[116] Y. A. Altay, A. S. Kremlev and A. A. Margun, "ECG Signal Filtering Approach for Detection of P, QRS, T Waves and Complexes in Short Single-Lead Recording," in Proc. 2019 IEEE Conference of Russian Young Researchers in Electrical and Electronic Engineering (EIConRus), Saint Petersburg and Moscow, Russia, 2019, pp. 1135-1140.

[117] V. Krasteva, R. Leber, I. Jekova, R. Schmid and R. Abächerli, "Classification of supraventricular and ventricular beats by QRS template matching and decision tree," in Proc. Computing in Cardiology 2014, Cambridge, MA, 2014, pp. 349-352.

[118] Y. Nakai, S. Izumi, M. Nakano, K. Yamashita, T. Fujii, H. Kawaguchi, and M. Yoshimoto, "Noise tolerant QRS detection using template matching with short-term autocorrelation," in Proc. 2014 36th Annual International Conference of the IEEE Engineering in Medicine and Biology Society, Chicago, IL, 2014, pp. 34-37.

[119] R. S. Andersen, E. S. Poulsen and S. Puthusserypady, "A novel approach for automatic detection of Atrial Fibrillation based on Inter Beat Intervals and Support Vector Machine," in Proc. 2017 39th Annual International Conference of the IEEE Engineering in Medicine and Biology Society (EMBC), Seogwipo, 2017, pp. 2039-2042.

[120] Y. Maki, Y. Monno, K. Yoshizaki, M. Tanaka and M. Okutomi, "Inter-Beat Interval Estimation from Facial Video Based on Reliability of BVP Signals," in Proc. 2019 41st Annual International Conference of the IEEE Engineering in Medicine and Biology Society (EMBC), Berlin, Germany, 2019, pp. 6525-6528.

[121] L. Mirmohamadsadeghi, S. Fallet, V. Moser, F. Braun and J. Vesin, "Real-time respiratory rate estimation using imaging photoplethysmography inter-beat intervals," in Proc. 2016 Computing in Cardiology Conference (CinC), Vancouver, BC, 2016, pp. 861-864.

[122] A. Al-Fuqaha, M. Guizani, M. Mohammadi, M. Aledhari, and M. Ayyash, "Internet of things: A survey on enabling technologies, protocols, and applications,” IEEE Commun. Surv. Tutorials, vol. 17, no. 4, pp. 2347-2376, 2015.

[123] R. Mahmoud, T. Yousuf, F. Aloul, and I. Zualkernan, "Internet of things (IoT) security: Current status, challenges and prospective measures," in Proc. 2015 10th International Conference for Internet Technology and Secured Transactions (ICITST), 2015, pp. 336-341.

[124] H. Wang and A. O. Fapojuwo, "A survey of enabling technologies of low power and long range machine-to-machine communications,” IEEE Commun. Surveys Tuts., vol. 19, no. 4, pp. 2621-2639, 2017.

[125] G. A. Akpakwu, B. J. Silva, G. P. Hancke, and A. M. Abu-Mahfouz, "A survey on 5G networks for the Internet of Things: Communication technologies and challenges,” IEEE Access, vol. 6, pp. 3619-3647, 2017.

[126] M. Agiwal, A. Roy and N. Saxena, "Next generation 5G wireless networks: A comprehensive survey," IEEE Communications Surveys \& Tutorials, vol. 18, no. 3, pp. 1617-1655, 2016.

[127] A. Koutsaftis, R. Kumar, P. Liu and S. S. Panwar, "Fast Inter-Base Station Ring (FIBR): A New Millimeter Wave Cellular Network Architecture," IEEE Journal on Selected Areas in Communications, vol. 37, no. 12, pp. 2699-2714, Dec. 2019.

[128] M. Bansal and B. Gandhi, "IoT \& Big Data in Smart Healthcare (ECG Monitoring)," in Proc. 2019 International Conference on Machine Learning, Big Data, Cloud and Parallel Computing (COMITCon), Faridabad, India, 2019, pp. 390-396.

[129] G. Xu, "IoT-assisted ECG monitoring framework with secure data transmission for health care applications," IEEE Access, 8, pp. 74586-74594

[130] F. Porée, G. Kervio, and G. Carrault, "ECG biometric analysis in different physiological recording conditions," Signal, Image and Video Process., vol. 10, no. 2, pp. 267-276, 2016.

[131] S. Wahabi, S. Pouryayevali, S. Hari, and D. Hatzinakos, "On evaluating ECG biometric systems: Session-dependence and body posture,” IEEE Trans. Inf. Forensics Security, vol. 9, no. 11, pp. 2002-2013, Nov. 2014.

[132] S. Pathoumvanh, S. Airphaiboon, and K. Hamamoto, "Robustness study of ECG biometric identification in heart rate variability conditions," IEEJ Trans. Elect. Electron. Eng., vol. 9, no. 3, pp. 294-301, May 2014.

[133] Deep speech: Scaling up end-to-end speech recognition. [Online]. Available: https://arxiv.org/abs/1412.5567.

[134] Q. Zhang, D. Zhou, and X. Zeng, "PulsePrint: Single-arm-ECG biometric human identification using deep learning," in Proc. IEEE 8th Annu. Ubiquitous Comput., Electron. Mobile Commun. Conf. (UEMCON), Oct. 2017, pp. 452456.

[135] A. Rizwan, A. Zoha, I. Mabrouk, H. Sabbour, A. Al-Sumaiti A. Alomaniy, M. Imran, and Q. Abbasi, “A review on the state of the art in atrial fibrillation detection enabled by machine learning," IEEE Reviews in Biomedical Engineering, 2020.

[136] S. S. Abdeldayem and T. Bourlai, "A Novel Approach for ECG-Based Human Identification Using Spectral Correlation and Deep Learning," IEEE Transactions on Biometrics, Behavior, and Identity Science, vol. 2, no. 1, pp. 1-14, Jan. 2020. 


\section{BIOGRAPHY OF AUTHORS}
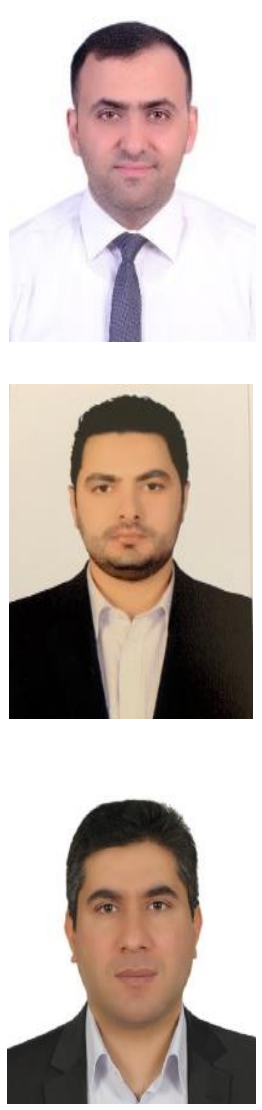

AHMET E. TOPCU received a BSc degree from the Department of Electrical and Electronics Engineering, Middle East Technical University, Ankara, Turkey in 1997; MSc degree in computer engineering from Syracuse University, Syracuse, NY, USA, in 2001; and PhD degree in computer science from Indiana University, Bloomington, IN, USA, in 2010. He is currently an Assistant Professor in the College of Engineering and Technology at American University of the Middle East, Kuwait. He is a member of IEEE and his current research interests include distributed systems, grid computing, cloud computing, big data, IoT, and blockchain.

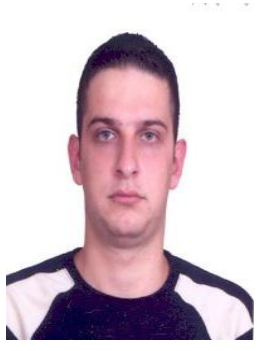

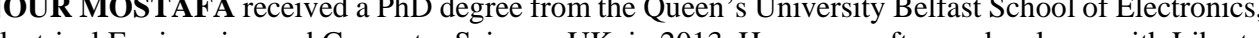
Engineering and Computer Science, UK, in 2013. He was a software developer with Liberty Technology, UK. He is currently an Associate Professor of Computer Science with the College of Engineering and Technology, American University of the Middle East. His current research interests include cloud, fog, and IoT computing; grid computing; large database management; artificial intelligence; and distributed computing.

Engineering Department, South Korea, in 2018. He was a research assistant in the Inje University Software Engineering Department, South Korea. He is currently an Assistant Professor of Computer Science with the College of Engineering and Technology, American University of the Middle East. His current research interests include IoT infrastructure and architecture, ECG signal processing, bioinformatics, real-time processing, human activities recognition, and E-health systems.

LOUAI SAKER received a master degree in networking at UPMC (Paris 6) and Telecom ParisTech (ENST), Paris, France, and a PhD degree in electronics and telecommunications from Telecom SudParis (INT) after three years of research work at Orange Labs. His research interests are in the areas of green networking, focusing on reducing the energy consumption of mobile networks. He is currently an Associate Professor in the Telecommunications and Networking Technology Department at American University of the Middle East. 